\title{
Characterization of the Asymptotic Teichmüller Space of the Open Unit Disk Through Shears
}

\author{
JINHUA FAN* AND JUN HU**
}

\begin{abstract}
We give a parametrization to the asymptotic Teichmüller space $A T(\mathbb{D})$ of the open unit disk $\mathbb{D}$ through equivalence classes of shear functions induced by quasisymmetric homeomorphisms on the Farey tessellation of $\mathbb{D}$. Then using the parametrization, we define a new metric on $A T(\mathbb{D})$. Two other related metrics are also introduced on $A T(\mathbb{D})$ by using cross-ratio distortions or quadrilateral dilatations under the boundary maps on degenerating sequences of quadruples or quadrilaterals. We show that the topologies induced by the three metrics are equivalent to the one induced by the Teichmüller metric on $A T(\mathbb{D})$. Before proving our main results, we revisit and rectify a mistake in the proof in [21] on the characterization of quasisymmetric homeomorphisms in terms of shear functions.
\end{abstract}

Keywords: Teichmüller space, asymptotic Teichmüller space, extremal maximal dilatation, maximal quadrilateral dilatation, shear.

\section{Introduction}

Let $\mathbb{D}$ be the open unit disk in the complex plane and centered at the origin, and let $\mathbb{S}^{1}$ be the boundary of $\mathbb{D}$. Denote by $T(\mathbb{D})$ the universal Teichmüller space and $A T(\mathbb{D})$ the asymptotic Teichmüller space of $\mathbb{D}$. Recently, two characterizations of the Teichmüller topology on $T(\mathbb{D})$ are given in [18] and [21]: one uses a uniform weak* topology defined on the Thurston parametrization of $T(\mathbb{D})$ ([23] or see elaborations and further investigations in [7], [10], and $[20]$ ) - the space of all Thurston-bounded measured geodesic laminations $\mathcal{L}$

Received December 8, 2013.

2000 Mathematics Subject Classification. 30C75, 30F60.

*This work is supported by NNSF of China (No. 11201228, No.11371194).

** The work is partially supported by PSC-CUNY grants and Brooklyn College Provost's Office for reassigned time in Spring 2013. 
on $\mathbb{D}$; the other is comprised of a parametrization of $T(\mathbb{D})$ by shear functions $s$ induced by quasisymmetric homeomorphisms of $\mathbb{S}^{1}$ on the Farey tessellation of $\mathbb{D}$ and a metric defined on the parametrization. In the following, by a topological characterization of $A T(\mathbb{D})$ we mean a parametrization of $A T(\mathbb{D})$ and a topology or metric on the parametrization equivalent to or with the induced topology equivalent to the Teichmüller topology on $A T(\mathbb{D})$. Very recently, a topological characterization of $A T(\mathbb{D})$ is given in [6] by using an asymptotically uniform weak* topology on a parametrization of $A T(\mathbb{D})$ by equivalence classes of laminations $\mathcal{L}$ in the Thurston parametrization of $T(\mathbb{D})$. In this paper, we give a topological characterization of $A T(\mathbb{D})$ by introducing a metric on a parametrization of $A T(\mathbb{D})$ by equivalence classes of the shear functions representing $T(\mathbb{D})$.

There are different models for $T(\mathbb{D})$ and $A T(\mathbb{D})$. In this paper, the main model we use for $T(\mathbb{D})$ is the collection $Q S\left(\mathbb{S}^{1}\right)$ of all quasisymmetric homeomorphisms of $\mathbb{S}^{1}$ fixing three points $1,-1$ and $i$; correspondingly, the one we use for $A T(\mathbb{D})$ is the quotient space $S\left(\mathbb{S}^{1}\right) \backslash Q S\left(\mathbb{S}^{1}\right)$, where $S\left(\mathbb{S}^{1}\right)$ is the collection of all symmetric homeomorphisms in $Q S\left(\mathbb{S}^{1}\right)$. Before we give the statements of our theorems, we first recall some background.

Consider $\mathbb{D}$ as the hyperbolic plane. The Farey tessellation $\mathcal{F}$ of $\mathbb{D}$ is a locally finite ideal triangulation of $\mathbb{D}$ that is invariant under the group of the isometries of $\mathbb{D}$ generated by the reflections in the hyperbolic metric with respect to the geodesics of $\mathcal{F}$ (see Section 4 for the details). The endpoints of the geodesics in $\mathcal{F}$ are called the tips of $\mathcal{F}$ and we denote the collection of all tips by $\mathcal{P}$. Each homeomorphism $h$ of $\mathbb{S}^{1}$ induces a real-valued function $s_{h}: \mathcal{F} \rightarrow \mathbb{R}$ as follows, which is called the shear function or coordinate of $h$. Given each edge $e \in \mathcal{F}$, let $\left(\Delta, \Delta_{1}\right)$ be the pair of the two adjacent triangles of $\mathcal{F}$ sharing a common boundary at $e$. Then $s_{h}(e)$ is defined to be the shear of the image pair $\left(h(\Delta), h\left(\Delta_{1}\right)\right)$, that is the signed hyperbolic distance between the orthogonal projections of the third vertices of $h(\Delta)$ and $h\left(\Delta_{1}\right)$ to their common edge (see Section 4 for its interpretation through the crossratio distortion under $h$ on a quadruple of four points on $\mathbb{S}^{1}$ ).

The shear norm of a homeomorphism $h$ of $\mathbb{S}^{1}$ is defined as follows. Let $\left\{e_{n}^{p}\right\}_{n \in \mathbb{Z}}$ be a fan in $\mathcal{F}$ with tip $p$ (see Section 4 for the definition), and let $s_{n}^{p}=s_{h}\left(e_{n}^{p}\right)$. For each $m, k \in \mathbb{Z}$ with $k \neq 0$, the following quantity $s(p ; m, k)$ can be interpreted as the cross-ratio distortion under $h$ on a quadruple of four points on $\mathbb{S}^{1}$ (see Section 4), which is defined as

$$
s(p ; m, k)=e^{s_{m}^{p}} \frac{1+e^{s_{m+1}^{p}}+e^{\left(s_{m+1}^{p}+s_{m+2}^{p}\right)}+\cdots+e^{\left(s_{m+1}^{p}+s_{m+2}^{p}+\cdots+s_{m+k}^{p}\right)}}{1+e^{-s_{m-1}^{p}}+e^{-\left(s_{m-1}^{p}+s_{m-2}^{p}\right)}+\cdots+e^{-\left(s_{m-1}^{p}+s_{m-2}^{p}+\cdots+s_{m-k}^{p}\right)}} .
$$


Then define

$$
M_{s}(p)=\sup _{m, k \in \mathbb{Z}}|\log s(p ; m, k)| \text { and } M_{s}=\sup _{p \in \mathcal{P}} M_{s}(p) .
$$

We call $M_{s}$ the shear norm of $h$. We say that a circle homeomorphism $h$ of $\mathbb{S}^{1}$ has a finite shear norm if $M_{s}$ is finite.

It is stated in Theorem A of [21] that a real-valued function $s: \mathcal{F} \rightarrow \mathbb{R}$ is equal to the shear function $s_{h}$ of a quasisymmetric homeomorphism $h$ of $\mathbb{S}^{1}$ if and only if $s$ has a finite shear norm. Let $\mathcal{X}$ be the collection of all real-valued functions $s: \mathcal{F} \rightarrow \mathbb{R}$ with finite shear norms. Then $\mathcal{X}$ gives a parametrization of $T(\mathbb{D})$. Given two elements $s_{1}, s_{2} \in \mathcal{X}$, define

$$
M_{s_{1}, s_{2}}(p)=\sup _{m, k \in \mathbb{Z}}\left|\log \frac{s_{1}(p ; m, k)}{s_{2}(p ; m, k)}\right| \text { and } d_{S}\left(s_{1}, s_{2}\right)=\sup _{p \in \mathcal{P}} M_{s_{1}, s_{2}}(p) .
$$

Then $d_{S}$ defines a metric on $\mathcal{X}$, called the shear metric. In Theorem B of [21], Sarić showed that the parametrization $\mathcal{X}$ of $T(\mathbb{D})$ equipped with the metric $d_{S}$ is topologically equivalent to $T(\mathbb{D})$ under the Teichmüller metric $d_{T}$. More explicitly, he proved that a sequence of points converges to a point in $T(\mathbb{D})$ under $d_{T}$ if and only if the sequence of the corresponding shear functions converges to the shear function of the limiting point under $d_{S}$.

The first goal of this paper is to give a parametrization to the asymptotic Teichmüller space $A T(\mathbb{D})$ via equivalence classes of shear functions, define a new metric on the parametrization, and show that the parametrization with the metric is a topological characterization of $A T(\mathbb{D})$ under the Teichmüller metric.

We first introduce a pseudo metric $d_{A S}$ on $\mathcal{X}$ (defined at the beginning of Section 5). This pseudo metric introduces an equivalence relation on $\mathcal{X}$ when two elements $s$ and $s^{\prime}$ of $\mathcal{X}$ are defined to be equivalent if $d_{A S}\left(s, s^{\prime}\right)=0$. We denote by $[s]$ the equivalence class of $s$ and by $\mathcal{A X}$ the quotient space under this equivalent relation. Then $d_{A S}$ induces a metric on $\mathcal{A X}$, which is continued to be denoted by $d_{A S}$ and called the asymptotic shear metric on $\mathcal{A X}$. In the following two theorems, we show that $\mathcal{A X}$ is a parametrization of $A T(\mathbb{D})$ and $\left(\mathcal{A X}, d_{A S}\right)$ is topologically equivalent to $\left(A T(\mathbb{D}), d_{A T}\right)$, where $d_{A T}$ is the Teichmüller metric on $A T(\mathbb{D})$.

Theorem 1. Let $h$ and $h^{\prime}$ be two points in $T(\mathbb{D})$ and let $s$ and $s^{\prime}$ be the shear functions induced by $h$ and $h^{\prime}$ respectively. Then $[h]=\left[h^{\prime}\right]$ if and only if $d_{A S}\left(s, s^{\prime}\right)=0$, where $[h]$ and $\left[h^{\prime}\right]$ denote two points in the asymptotic Teichmüller space $A T(\mathbb{D})$. 
Theorem 2. Let $[h] \in A T(\mathbb{D})$ and let $\left\{\left[h_{n}\right]\right\}_{n=1}^{\infty}$ be a sequence of points in $A T(\mathbb{D})$. Assume that $s_{n}$ and $s$ are the shear functions induced by $h_{n}$ and $h$ respectively. Then $d_{A T}\left(\left[h_{n}\right],[h]\right) \rightarrow 0$ if and only if $d_{A S}\left(\left[s_{n}\right],[s]\right) \rightarrow 0$.

The Douady-Earle extensions [2] of circle homeomorphisms of $\mathbb{S}^{1}$ have played an important role in the study of Teichmüller spaces and asymptotic Teichmüller spaces. For some recent applications of these extensions, we refer to [5], 6], [17], [18], and [21]. Further investigation on regularities of the Douady-Earle extensions of circle homeomorphisms or generalizations of this kind of extensions to large classes of circle continuous maps can be found in [11]- 15$]$ and [16].

The conformal naturality and one local regularity near the origin of the Douady-Earle extensions together play a crucial role in the proof of Theorem $\mathrm{A}$ in [21. That local regularity is summarized as Lemma 2.2 in [21], which states as follows. Let $\widehat{\mathbb{R}}=\mathbb{R} \cup\{\infty\}$ be the extended real line, which represents the boundary of the hyperbolic plane when it takes the upper half plane $\mathbb{H}$ as a model. Let $\left\{h_{n}\right\}_{n=1}^{\infty}$ be a sequence of orientation-preserving homeomorphisms $h_{n}$ of $\widehat{\mathbb{R}}$ fixing three points 0,1 and $\infty$, and let $\mu_{n}$ be the Beltrami coefficient of the Douady-Earle extension ex $\left(h_{n}\right)$ of $h_{n}$ for each $n$. If there exists $c_{0} \geq 1$ such that

$$
-c_{0} \leq h_{n}(-1) \leq-\frac{1}{c_{0}}
$$

for all $n$, then there exists a neighborhood $U$ of the imaginary unit $i \in \mathbb{H}$ and a constant $0<c<1$ such that

$$
\left.|| \mu_{n}\right|_{U} \|_{\infty} \leq c<1
$$

for all $n$.

Theorem A of [21] gives a necessary and sufficient condition for a function $s: \mathcal{F} \rightarrow \mathbb{R}$ to be induced by a quasisymmetric (resp. symmetric) homeomorphism of $\mathbb{S}^{1}$. The necessity of the condition can be easily obtained, see Remark 1 in Section 4 (resp. Remark 2 in Section 5). The main part of the proof of Theorem A is to show that $M_{s}<\infty$ is sufficient for $s$ to be induced by a quasisymmetric homeomorphism of $\mathbb{S}^{1}$. This part is proved there by applying the above local regularity of Douady-Earle extensions, that is Lemma 2.2 in [21]. Counter-examples have been found for that lemma. Using an algorithm called the MAY operator [1, the first counter-example was tested numerically and then proved in $[13$. In Section 3 of this paper, we provide a slightly different example without involving any explicit formulas so that it can be verified relatively easily. This means that there is a mistake 
or gap in [21] in the proof of the sufficient condition for $s$ to be induced by a quasisymmetric homeomorphism. The second goal of this paper is to rectify that mistake or bridge the gap in the proof caused by the counter-examples. We thereafter develop in Section 3 a new lemma for being used to bridge the gap. Then in Section 4, we apply our new lemma to show how the gap is bridged by proving an improved version of the sufficient condition as follows.

Theorem 3. Let $h$ be a homeomorphism of $\widehat{\mathbb{R}}$ and let $s$ be the shear function induced by $h$. Then

$$
K(e x(h))<C\left(M_{s}\right) ;
$$

that is, the maximal dilatation of the Douady-Earle extension of $h$ is bounded from above by a positive constant only depending on the shear norm of $h$.

In the course of developing and proving our Theorems 1 and 2 , two related metrics on $A T(\mathbb{D})$ come into place naturally: one is defined by using cross-ratio distortions under the boundary maps on degenerating sequences of quadruples and the other is defined by using quadrilateral dilatations under the boundary maps on degenerating sequences of quadrilaterals. Tracing back to similar metrics introduced on the universal Teichmüller space $T(\mathbb{D})$, in the remaining part of this introduction section we recall the two metrics on $T(\mathbb{D})$ defined by using cross-ratio distortions and quadrilateral dilatations respectively and then define the corresponding metrics on $A T(\mathbb{D})$.

For each $h \in T(\mathbb{D})$, let

$$
K_{e}(h)=\inf _{\left.f\right|_{\mathbb{S} 1}=h} K(f),
$$

where the infimum is taken over all quasiconformal extensions $f$ of $h$ to $\mathbb{D}$. Then the Teichmüller metric $d_{T}$ is defined as

$$
d_{T}\left(h_{1}, h_{2}\right)=\frac{1}{2} \log K_{e}\left(h_{2} \circ h_{1}^{-1}\right)
$$

for any two points $h_{1}, h_{2} \in T(\mathbb{D})$.

Given a quadruple $Q=\{a, b, c, d\}$ of four distinct points $a, b, c$ and $d$ on $\mathbb{S}^{1}$ arranged in counterclockwise order, let $M(Q)$ denote the conformal modulus of the (topological) quadrilateral $\mathbb{D}(a, b, c, d)$. Each orientationpreserving homeomorphism $h$ of $\mathbb{S}^{1}$ maps $\mathbb{D}(a, b, c, d)$ to another quadrilateral $\mathbb{D}(h(a), h(b), h(c), h(d))$. Correspondingly, we let $M(h(Q))$ denote the conformal modulus of $\mathbb{D}(h(a), h(b), h(c), h(d))$. Given an element $h \in T(\mathbb{D})$, 
the maximal quadrilateral dilatation of $h$ is defined as

$$
K_{m}(h)=\sup _{Q}\left\{\frac{M(h(Q))}{M(Q)}\right\},
$$

where the supremum is taken over all quadruples $Q$. Given any two points $h_{1}, h_{2} \in T(\mathbb{D})$, we define

$$
d_{M}\left(h_{1}, h_{2}\right)=\frac{1}{2} \log K_{m}\left(h_{2} \circ h_{1}^{-1}\right) .
$$

By definition, it is clear that

$$
d_{M}\left(h_{1}, h_{2}\right)=\frac{1}{2} \sup _{Q}\left|\log \frac{M\left(h_{2}(Q)\right)}{M\left(h_{1}(Q)\right)}\right| .
$$

It is well known that

$$
\frac{1}{K(f)} \leq \frac{M(h(Q))}{M(Q)} \leq K(f)
$$

for any quasiconformal extension $f$ of $h$ and any quadruple $Q$. It follows that for any $h \in T(\mathbb{D})$,

$$
K_{m}(h) \leq K_{e}(h),
$$

and for any two points in $h_{1}, h_{2} \in T(\mathbb{D})$,

$$
d_{M}\left(h_{1}, h_{2}\right) \leq d_{T}\left(h_{1}, h_{2}\right) .
$$

Given a homeomorphism $h$ of $\mathbb{S}^{1}$ and a quadruple $Q=\{a, b, c, d\}$ of four distinct points on $\mathbb{S}^{1}$ arranged in the counterclockwise order, let $h(Q)$ be the image quadruple $\{h(a), h(b), h(c), h(d)\}$ and let $\operatorname{cr}(Q)$ denote the cross ratio of $Q$ defined by

$$
c r(Q)=\frac{(b-a)(d-c)}{(c-b)(d-a)} .
$$

Given any two points $h_{1}, h_{2} \in T(\mathbb{D})$, we define

$$
d_{C}\left(h_{1}, h_{2}\right)=\sup _{c r(Q)=1}\left|\log \frac{c r\left(h_{2}(Q)\right)}{c r\left(h_{1}(Q)\right)}\right| .
$$

One has known that $d_{M}$ and $d_{C}$ are metrics on $T(\mathbb{D})$ and the three metrics $d_{T}, d_{M}$ and $d_{C}$ on $T(\mathbb{D})$ are topologically equivalent. Since we cannot 
find references explicitly giving these two statements, we state them as the following folklore theorems and provide their proofs in Section 2. The proofs are not long.

Theorem 4 (Folklore Theorem 1). Both $d_{M}$ and $d_{C}$ define metrics on $T(\mathbb{D})$.

Theorem 5 (Folklore Theorem 2). The three metrics $d_{T}, d_{M}$ and $d_{C}$ on $T(\mathbb{D})$ are topologically equivalent.

An orientation-preserving homeomorphism $h$ of $\mathbb{S}^{1}$ is said to be symmetric if

$$
(1+\delta(x, t))^{-1} \leq \frac{|h(x+t)-h(x)|}{|h(x)-h(x-t)|} \leq 1+\delta(x, t),
$$

where $\delta(x, t) \rightarrow 0$ uniformly for all $x \in \mathbb{S}^{1}$ as $t \rightarrow 0$. By letting $T_{0}(\mathbb{D})$ be the subspace of $T(\mathbb{D})$ whose elements are symmetric homeomorphisms, the asymptotic Teichmüller space can be expressed as the quotient space

$$
A T(\mathbb{D})=T_{0}(\mathbb{D}) \backslash T(\mathbb{D}) .
$$

Given any $h \in T(\mathbb{D})$, we denote by $[h]$ the corresponding point in $A T(\mathbb{D})$. This space was studied by Gardiner and Sullivan in [9] and the asymptotic Teichmüller spaces of Riemann surfaces were studied in [3], [4], [5], 8] and etc.

Parallel to $d_{T}, d_{M}$ and $d_{C}$ on $T(\mathbb{D})$, three metrics can be defined on $A T(\mathbb{D})$.

Let $f$ be a quasiconformal mapping on $\mathbb{D}$. The boundary dilatation $H(f)$ of $f$ is defined as

$$
H(f)=\inf _{E} K\left(\left.f\right|_{\mathbb{D} \backslash E}\right),
$$

where the infimum is taken over all compact subsets $E$ of $\mathbb{D}$. Given any point $[h] \in A T(\mathbb{D})$, the boundary dilatation of $[h]$ is defined as

$$
K_{a e}([h])=\inf _{\left.f\right|_{\mathbb{S}^{1}} \in[h]} H(f) .
$$

The Teichmüller distance between two points $\left[h_{1}\right],\left[h_{2}\right] \in A T(\mathbb{D})$ is defined as

$$
d_{A T}\left(\left[h_{1}\right],\left[h_{2}\right]\right)=\frac{1}{2} \log K_{a e}\left(\left[h_{2} \circ\left(h_{1}\right)^{-1}\right]\right) .
$$


Given a topological quadrilateral $Q=\mathbb{D}(a, b, c, d)$ or quadruple $Q=$ $\{a, b, c, d\}$, we define the minimal scale of $Q$ as

$$
s(Q)=\min \{|a-b|,|b-c|,|c-d|,|d-a|\} .
$$

Then a sequence $\left\{Q_{n}=\mathbb{D}\left(a_{n}, b_{n}, c_{n}, d_{n}\right)\right\}_{n=1}^{\infty}$ of topological quadrilaterals is said to be degenerating if $s\left(Q_{n}\right) \rightarrow 0$ as $n \rightarrow \infty$. A sequence $\left\{Q_{n}=\right.$ $\left.\left\{a_{n}, b_{n}, c_{n}, d_{n}\right\}\right\}_{n=1}^{\infty}$ of quadruples is said to be degenerating if $\operatorname{cr}\left(Q_{n}\right)=1$ for each $n$ and $s\left(Q_{n}\right) \rightarrow 0$ as $n \rightarrow \infty$. For any $[h] \in A T(\mathbb{D})$, we define the asymptotically maximal quadrilateral dilatation of $[h]$ as

$$
K_{a m}([h])=\sup _{\left\{Q_{n}\right\}} \limsup _{n \rightarrow \infty}\left\{\frac{M\left(h\left(Q_{n}\right)\right)}{M\left(Q_{n}\right)}\right\},
$$

where the supremum is taken over all degenerating sequences $\left\{Q_{n}\right\}_{n=1}^{\infty}$ of topological quadrilaterals. Given any two points $\left[h_{1}\right],\left[h_{2}\right] \in A T(\mathbb{D})$, we define

$$
d_{A M}\left(\left[h_{1}\right],\left[h_{2}\right]\right)=\frac{1}{2} \log K_{a m}\left(\left[h_{2} \circ h_{1}^{-1}\right]\right) .
$$

Clearly,

$$
d_{A M}\left(\left[h_{1}\right],\left[h_{2}\right]\right)=\frac{1}{2} \sup _{\left\{Q_{n}\right\}} \limsup _{n \rightarrow \infty}\left|\log \frac{M\left(h_{2}\left(Q_{n}\right)\right)}{M\left(h_{1}\left(Q_{n}\right)\right)}\right|,
$$

where the supremum is taken over all degenerating sequences $\left\{Q_{n}\right\}_{n=1}^{\infty}$ of topological quadrilaterals.

From the proof of Theorem 1 of $[25$, it follows that for any $[h] \in A T(\mathbb{D})$,

$$
K_{a e}([h]) \geq K_{a m}([h]) .
$$

Thus, for any two points $\left[h_{1}\right],\left[h_{2}\right] \in A T(\mathbb{D})$,

$$
d_{A M}\left(\left[h_{1}\right],\left[h_{2}\right]\right) \leq d_{A T}\left(\left[h_{1}\right],\left[h_{2}\right]\right) .
$$

Given any two points $\left[h_{1}\right],\left[h_{2}\right] \in A T(\mathbb{D})$, we define

$$
d_{A C}\left(\left[h_{1}\right],\left[h_{2}\right]\right)=\sup _{\left\{Q_{n}\right\}} \limsup _{n \rightarrow \infty}\left|\log \frac{c r\left(h_{2}\left(Q_{n}\right)\right)}{\operatorname{cr}\left(h_{1}\left(Q_{n}\right)\right)}\right|,
$$

where the supremum is taken over all degenerating sequences $\left\{Q_{n}\right\}_{n=1}^{\infty}$ of quadruples.

In Section 2, we also show the following two theorems. 
Characterization of Asymptotic Teichmüller Space Through Shears 521

Theorem 6. Both $d_{A M}$ and $d_{A C}$ define metrics on $A T(\mathbb{D})$.

Theorem 7. The three metrics $d_{A T}, d_{A M}$ and $d_{A C}$ on $A T(\mathbb{D})$ are topologically equivalent.

The paper is arranged as follows. We first give proofs of Theorems 4-7 in the second section. In the third section, we provide a counter-example to Lemma 2.2 in [21] and introduce a new lemma. Then in the fourth section, we apply the new lemma to bridge the gap in the proof of Theorem A in 21] by showing Theorem 3 . The proofs of Theorems 1 and 2 are given in the fifth section.

Acknowledgement: The authors wish to thank Professor Frederick Gardiner and the referee for their comments and suggestions on improving the writing of the paper.

\section{Topological equivalence of three metrics on $T(\mathbb{D})$ and} $A T(\mathbb{D})$

In this section, we show Theorems 4-7.

Let $\rho_{(0,-1)}$ be the Poincaré density on $\mathbb{C} \backslash\{0,-1\}$ and let $d_{\rho_{(0,-1)}}(\alpha, \beta)$ be the distance under $\rho_{(0,-1)}$ between two points $\alpha, \beta \in \mathbb{C} \backslash\{0,-1\}$. The following result of [24] is applied to show Theorem 4 .

Lemma 1. 24 For any two quadruples $Q_{1}$ and $Q_{2}$ of four distinct points on $\mathbb{S}^{1}$ arranged in counterclockwise order,

$$
d_{\rho_{(0,-1)}}\left(\operatorname{cr}\left(Q_{1}\right), \operatorname{cr}\left(Q_{2}\right)\right)=\left|\log \frac{M\left(Q_{1}\right)}{M\left(Q_{2}\right)}\right| .
$$

Proof of Theorem 4. It is easy to check that $d_{M}$ and $d_{C}$ are symmetric and satisfy the triangle inequality. To prove $d_{M}$ and $d_{C}$ to be metrics, we only need to check that $d_{M}\left(h_{1}, h_{2}\right)=0$ or $d_{C}\left(h_{1}, h_{2}\right)=0$ implies $h_{1}=h_{2}$.

We first show that $d_{M}\left(h_{1}, h_{2}\right)=0$ implies $d_{C}\left(h_{1}, h_{2}\right)=0$. If on the contrary, $d_{C}\left(h_{1}, h_{2}\right)>0$, then there exist $m>0$ and a quadruple $Q=$ $\{a, b, c, d\} \subset \mathbb{S}^{1}$ with $\operatorname{cr}(Q)=1$ such that

$$
\frac{c r\left(h_{1}(Q)\right)}{c r\left(h_{2}(Q)\right)}>1+m .
$$

Then Lemma 1 implies

$$
\left|\log \frac{M\left(h_{1}(Q)\right)}{M\left(h_{2}(Q)\right)}\right|>0,
$$


which is a contradiction to $d_{M}\left(h_{1}, h_{2}\right)=0$.

Now we show that $d_{C}\left(h_{1}, h_{2}\right)=0$ implies $h_{1}=h_{2}$. Let $A(z)=-\frac{z-i}{z+i}$, which is a Möbius transformation from $\mathbb{H}$ to $\mathbb{D}$, and let $\tilde{h}_{1}=A^{-1} \circ h_{1} \circ A$ and $\tilde{h}_{2}=A^{-1} \circ h_{2} \circ A$. Then $d_{C}\left(h_{1}, h_{2}\right)=0$ means $\operatorname{cr}\left(\tilde{h}_{1}(Q)\right)=\operatorname{cr}\left(\tilde{h}_{2}(Q)\right)$ for any quadruple $Q=\{a, b, c, d\} \subset \widehat{\mathbb{R}}$ with $\operatorname{cr}(Q)=1$. Since $\tilde{h}_{1}$ and $\tilde{h}_{2}$ fix 0,1 and $\infty$, by applying the condition $\operatorname{cr}\left(\tilde{h}_{1}(Q)\right)=\operatorname{cr}\left(\tilde{h}_{2}(Q)\right)$ repeatedly on appropriate quadruples $Q \subset \mathbb{Z}$, we first obtain that $\tilde{h}_{1}(x)=\tilde{h}_{2}(x)$ for all $x \in \mathbb{Z}$. Then by using quadruples $Q \subset \frac{\mathbb{Z}}{2}$, we conclude that $\tilde{h}_{1}(x)=\tilde{h}_{2}(x)$ for all $x \in \frac{\mathbb{Z}}{2}$. Inductively, $\tilde{h}_{1}(x)=\tilde{h}_{2}(x)$ for all $x \in \frac{\mathbb{Z}}{2^{n}}$ for each $n \in \mathbb{N}$. Since $\cup_{n=1}^{\infty} \frac{\mathbb{Z}}{2^{n}}$ is dense in $\widehat{\mathbb{R}}$, it follows that $\tilde{h}_{1}(x)=\tilde{h}_{2}(x)$ for all $x \in \widehat{\mathbb{R}}$. Thus, $h_{1}=h_{2}$.

From now on, the so-called Douady-Earle extensions ex(h) ([2]) of circle homeomorphisms $h$ will be frequently used in arguments to prove our theorems. These extensions are remarkable in the sense that for any two conformal isometries $A$ and $\gamma$ of $\mathbb{D}$,

$$
\operatorname{ex}(A \circ h \circ \gamma)=A \circ \operatorname{ex}(h) \circ \gamma,
$$

where $A \circ h \circ \gamma$ stands for the circle homeomorphism given by $\left.A\right|_{\mathbb{S}^{1}} \circ h \circ$ $\left.\gamma\right|_{\mathbb{S}^{1}}$. This property is called the conformal naturality of the Douady-Earle extension.

Let us recall the definition of $\operatorname{ex}(h)$. Given a point $z \in \mathbb{D}$, let $\eta_{z}$ be the harmonic measure on $\mathbb{S}^{1}$ viewed from $z$. Then $\operatorname{ex}(h)(z)$ is defined to be the conformal barycenter $B(\mu)$ of the pushforward measure $\mu=h_{*} \eta_{z}$ of $\eta_{z}$ under $h$, which is defined as a point $w \in \mathbb{D}$ such that

$$
V_{\mu}(w)=\left(1-|w|^{2}\right) \int_{\mathbb{S}^{1}} \frac{\zeta-w}{1-\bar{w} \zeta} d \mu(\zeta)=0 .
$$

The existence and uniqueness of $B(\mu)$ was proved in [2] by using PoincaréHopf Index Theorem. An elementary and direct proof was given in [14]. Clearly, for any $z \in \mathbb{S}^{1}, \operatorname{ex}(h)(z)$ is defined to be $h(z)$.

We also need the following properties of $e x(h)$ in this paper. Each extension $e x(h)$ is a homeomorphism of $\overline{\mathbb{D}}$ and is real-analytic on $\mathbb{D}$. Furthermore, if $h$ admits a quasiconformal extension to $\mathbb{D}$ then $e x(h)$ is also quasiconformal. The following property of the extension operator $e x$ is also very useful when these extensions are applied, which is Proposition 2 in [2].

Let $\mathcal{H}\left(\mathbb{S}^{1}\right)$ be the set of homeomorphisms of $\mathbb{D}$, which is a subset of the Banach space $\mathcal{B}\left(\mathbb{S}^{1}, \mathbb{C}\right)$ of complex-valued continuous functions on $\mathbb{S}^{1}$ with 
Characterization of Asymptotic Teichmüller Space Through Shears 523

the sup norm. Let $\mathcal{D}$ iff $(\mathbb{D}) \cap \mathcal{H}(\overline{\mathbb{D}})$ be the set of $\mathcal{C}^{\infty}$ diffeomorphisms of $\mathbb{D}$ with homeomorphic extensions to $\overline{\mathbb{D}}$, where $\operatorname{Diff}(\mathbb{D})$ and $\mathcal{H}(\overline{\mathbb{D}})$ are subsets of the vector spaces $\mathcal{C}^{\infty}(\mathbb{D}, \mathbb{C})$ and $\mathcal{B}(\overline{\mathbb{D}}, \mathbb{C})$ with their standard topologies respectively and the topology on $\mathcal{D}$ if $f(\mathbb{D}) \cap \mathcal{H}(\overline{\mathbb{D}})$ is induced by the diagonal embedding in $\mathcal{D}$ iff $(\mathbb{D}) \times \mathcal{H}(\overline{\mathbb{D}})$. Then the extension operator ex has the following property.

Proposition 1. [2] The extension operator

$$
e x: \mathcal{H}\left(\mathbb{S}^{1}\right) \rightarrow \mathcal{D} \text { iff }(\mathbb{D}) \cap \mathcal{H}(\overline{\mathbb{D}}): h \mapsto \operatorname{ex}(h)
$$

is continuous.

The following two results of $\mathrm{Hu}$ and Muzician show how the maximal dilatation of the extension $e x(h)$ is controlled by the cross-ratio distortion norm of $h$ globally or locally.

Proposition 2. [11] There exists a universal constant $C>0$ such that

$$
\log K(e x(h)) \leq C\|h\|_{c r},
$$

where $K(e x(h))$ is the maximal dilatation of ex $(h)$ and $\|h\|_{c r}$ is the crossratio distortion norm of $h$ defined as

$$
\|h\|_{c r}=\sup _{c r(Q)=1}|\log \operatorname{cr}(h(Q))|,
$$

where the supreme is taken over all quadruples $Q$ of four distinct points on $\mathbb{S}^{1}$ arranged in counterclockwise order.

Lemma 2. [12] Let $p \in \mathbb{S}^{1}$ and $I_{p}$ be an open arc on $\mathbb{S}^{1}$ containing $p$ and symmetric with respect to $p$. Assume that

$$
\left\|\left.h\right|_{I_{p}}\right\|_{c r}=\sup _{\operatorname{cr}(Q)=1}|\log \operatorname{cr}(h(Q))|<\infty,
$$

where the supremum is taken over all quadruples $Q \subset I_{p}$. Then there exists an open hyperbolic half plane $U_{p}$ with $p$ at the middle of its boundary on $\mathbb{S}^{1}$ such that

$$
\log K\left(e x(h) \mid U_{p}\right) \leq C_{1}\left\|\left.h\right|_{I_{p}}\right\|_{c r}+C_{2}
$$

for two universal positive constants $C_{1}$ and $C_{2}$, where $K\left(e x(h) \mid U_{p}\right)$ is the maximal dilatation of ex $(h)$ on $U_{p}$. 
Now we are ready to show Theorems 5-7.

Proof of Theorem 5. Let $h \in T(\mathbb{D})$ and let $\left\{h_{n}\right\}_{n=1}^{\infty}$ be a sequence of points in $T(\mathbb{D})$.

At first, since $d_{M}\left(h_{n}, h\right) \leq d_{T}\left(h_{n}, h\right)$, it follows that $d_{T}\left(h_{n}, h\right) \rightarrow 0$ implies $d_{M}\left(h_{n}, h\right) \rightarrow 0$ as $n \rightarrow \infty$.

Secondly, we show that $d_{M}\left(h_{n}, h\right) \rightarrow 0$ implies $d_{C}\left(h_{n}, h\right) \rightarrow 0$ as $n \rightarrow \infty$. If on the contrary this is not true, then there exists a subsequence of $\left\{h_{n}\right\}_{n=1}^{\infty}$, denoted again by $\left\{h_{n}\right\}_{n=1}^{\infty}$ for simplicity, such that $d_{C}\left(h_{n}, h\right)>\varepsilon_{0}$ for all $n$ and some $\varepsilon_{0}>0$. Thus there is a sequence $\left\{Q_{n}=\left\{a_{n}, b_{n}, c_{n}, d_{n}\right\}\right\}_{n=1}^{\infty} \subset \mathbb{S}^{1}$ of quadruples with $\operatorname{cr}\left(Q_{n}\right)=1$ such that for all $n$,

$$
\frac{c r\left(h_{n}\left(Q_{n}\right)\right)}{\operatorname{cr}\left(h\left(Q_{n}\right)\right)}>e^{\varepsilon_{0}} .
$$

Since $h$ is a given quasisymmetric homeomorphism and $\operatorname{cr}\left(Q_{n}\right)=1$, $\left\{\operatorname{cr}\left(h\left(Q_{n}\right)\right)\right\}_{n=1}^{\infty}$ stays in a compact subset of the positive half real axis. Thus, there exists $\varepsilon_{0}^{\prime}>0$ such that for all $n$,

$$
d_{\rho_{(0,-1)}}\left(\operatorname{cr}\left(h_{n}\left(Q_{n}\right), \operatorname{cr}\left(h\left(Q_{n}\right)\right)\right)>\varepsilon_{0}^{\prime}\right.
$$

Using Lemma 1, we conclude that for all $n$,

$$
\left|\log \frac{M\left(h_{n}\left(Q_{n}\right)\right)}{M\left(h\left(Q_{n}\right)\right)}\right|>\varepsilon_{0}^{\prime} .
$$

This is a contradiction to the assumption that $d_{M}\left(h_{n}, h\right) \rightarrow 0$ as $n \rightarrow \infty$.

Finally, we show that $d_{C}\left(h_{n}, h\right) \rightarrow 0$ implies $d_{T}\left(h_{n}, h\right) \rightarrow 0$ as $n \rightarrow \infty$. If on the contrary this is not true, then by passing to a subsequence we may assume that $d_{T}\left(h_{n}, h\right)>\varepsilon_{1}$ for all $n$ and some $\varepsilon_{1}>0$.

Let $\operatorname{ex}\left(h_{n}\right)$ be the Douady-Earle extension of $h_{n}$. Since $d_{C}\left(h_{n}, h\right) \rightarrow 0$ as $n \rightarrow \infty$, there exists $C>0$ such that for all $n$ and any quadruple $Q$ with $\operatorname{cr}(Q)=1$,

$$
\left|\log c r\left(h_{n}(Q)\right)\right|<C+|\log c r(h(Q))| \leq C+\|\left. h\right|_{c r}<+\infty .
$$

Using Proposition 2, there exists $K_{0}>1$ such that for all $n$,

$$
K\left(e x\left(h_{n}\right)\right) \leq K_{0}
$$

Thus, $\left\{h_{n}\right\}$ is a bounded sequence in $T(\mathbb{D})$. Passing to subsequences, we may assume that $\left\{h_{n}\right\}_{n=1}^{\infty}$ converges to a quasisymmetric homeomorphism 
$h^{\prime}$ uniformly on $\mathbb{S}^{1}$ and also converges to a point $h^{\prime \prime}$ in $T(\mathbb{D})$ under the metric $d_{T}$. Note that $h^{\prime}$ and $h^{\prime \prime}$ fix the same three points as each $h_{n}$ does. Using the conclusions in the previous two steps, we obtain $d_{C}\left(h_{n}, h^{\prime \prime}\right) \rightarrow 0$ as $n \rightarrow \infty$. Then for each quadruple $Q$ with $\operatorname{cr}(Q)=1$,

$$
\operatorname{cr}\left(h^{\prime \prime}(Q)\right)=\lim _{n \rightarrow \infty} \operatorname{cr}\left(h_{n}(Q)\right)=\operatorname{cr}\left(h^{\prime}(Q)\right) .
$$

Therefore, $h^{\prime}=h^{\prime \prime}$. Since $d_{C}\left(h_{n}, h\right) \rightarrow 0$ as $n \rightarrow \infty$, we concluded that $h=h^{\prime}=h^{\prime \prime}$. Thus, $d_{T}\left(h_{n}, h\right) \rightarrow 0$ as $n \rightarrow \infty$. This is a contradiction to the assumption that $d_{T}\left(h_{n}, h\right)>\varepsilon_{1}$ for all $n$.

The proof is complete.

Proof of Theorem 6. Using definitions, it is easy to check that $d_{A M}$ and $d_{A C}$ are symmetric and satisfy the triangle inequality. To prove $d_{A M}$ and $d_{A C}$ to be metrics, it remains to show that $d_{A M}\left(\left[h_{1}\right],\left[h_{2}\right]\right)=0$ or $d_{A C}\left(\left[h_{1}\right],\left[h_{2}\right]\right)=0$ implies $\left[h_{1}\right]=\left[h_{2}\right]$.

Using the same argument to show that $d_{M}\left(h_{1}, h_{2}\right)=0$ implies $d_{C}\left(h_{1}, h_{2}\right)=0$ in the proof of Theorem 4 , one can see that $d_{A M}\left(\left[h_{1}\right],\left[h_{2}\right]\right)=$ 0 implies $d_{A C}\left(\left[h_{1}\right],\left[h_{2}\right]\right)=0$.

In the following, we apply some properties of Douady-Earle extensions to show that $d_{A C}\left(\left[h_{1}\right],\left[h_{2}\right]\right)=0$ implies $\left[h_{1}\right]=\left[h_{2}\right]$. Suppose on the contrary that $\left[h_{1}\right] \neq\left[h_{2}\right]$, which means $h_{1} \circ\left(h_{2}\right)^{-1}$ is not symmetric. Let $e x\left(h_{1}\right)$ and $e x\left(h_{2}\right)$ be the Douady-Earle extensions of $h_{1}$ and $h_{2}$ respectively. Using a result of [5], we know that $e x\left(h_{1}\right) \circ\left(e x\left(h_{2}\right)\right)^{-1}$ is not asymptotically conformal on $\mathbb{D}$, which means that there exist a constant $\varepsilon_{0}>0$ and a sequence $\left\{D_{n}\right\}_{n=1}^{\infty}$ of hyperbolic disks in $\mathbb{D}$ of diameter 1 with the Euclidean distance from $D_{n}$ to $\mathbb{S}^{1}$ approaching 0 as $n \rightarrow \infty$ such that

$$
\left\|\mu_{e x\left(h_{1}\right)}\left|D_{n}-\mu_{e x\left(h_{2}\right)}\right| D_{n}\right\|_{L^{\infty}} \geq \varepsilon_{0}
$$

for all $n$. Let $D_{0}$ be the hyperbolic disk on $\mathbb{D}$ of diameter 1 and centered at 0 , and assume that $\gamma_{n} \in M \ddot{o} b(\mathbb{D})$ and $\gamma_{n}\left(D_{0}\right)=D_{n}$, where $M \ddot{o} b(\mathbb{D})$ is the group of all Möbius transformation preserving $\mathbb{D}$. Let $A_{1 n}$ and $A_{2 n} \in M \ddot{b} b(\mathbb{D})$ such that $A_{1 n} \circ h_{1} \circ \gamma_{n}$ and $A_{2 n} \circ h_{2} \circ \gamma_{n}$ fix $1,-1, i$ for each $n$.

Sublemma. Given any quadruple $Q=\{a, b, c, d\}$ of four points on $\mathbb{S}^{1}$ in counterclockwise order with $\operatorname{cr}(Q)=1, s\left(\gamma_{n}(Q)\right) \rightarrow 0$ as $n \rightarrow \infty$.

This sublemma can be proved as follows. Let $\beta$ be the common perpendicular geodesic segment to the geodesics connecting $a$ and $b$ and $c$ and $d$ respectively. Since the hyperbolic distance between the middle point of $\beta$ and the hyperbolic center of $D_{0}$ is preserved under the maps $\gamma_{n}$ and the 
Euclidean distance from $\gamma_{n}\left(D_{0}\right)$ to $\mathbb{S}^{1}$ approaches 0 as $n \rightarrow \infty$, it follows that both the Euclidean diameter of $\gamma_{n}\left(D_{0}\right)$ and the Euclidean length of $\gamma_{n}(\beta)$ approaches 0 as $n \rightarrow \infty$. Thus, $s\left(\gamma_{n}(Q)\right) \rightarrow 0$ as $n \rightarrow \infty$.

Using the previous sublemma and the assumption that $d_{A C}\left(\left[h_{1}\right],\left[h_{2}\right]\right)=$ 0 , we obtain

$$
\lim _{n \rightarrow \infty} \frac{c r\left(A_{1 n} \circ h_{1} \circ \gamma_{n}(Q)\right)}{c r\left(A_{2 n} \circ h_{2} \circ \gamma_{n}(Q)\right)}=1
$$

Let $e x\left(A_{1 n} \circ h_{1} \circ \gamma_{n}\right)$ and $e x\left(A_{2 n} \circ h_{2} \circ \gamma_{n}\right)$ be the Douady-Earle extensions of $A_{1 n} \circ h_{1} \circ \gamma_{n}$ and $A_{2 n} \circ h_{2} \circ \gamma_{n}$ respectively. Since these quasiconformal mappings fix three common points and have constant maximal dilatations, passing to subsequences, we may assume that they converge to two quasisymmetric homeomorphisms $\widetilde{h}_{1}$ and $\widetilde{h}_{2}$ uniformly on $\mathbb{S}^{1}$. Using $(2.3)$ and the convergence of the two sequences, we obtain

$$
\operatorname{cr}\left(\widetilde{h}_{1}(Q)\right)=\operatorname{cr}\left(\widetilde{h}_{2}(Q)\right)
$$

for any quadruple $Q \in \mathbb{S}^{1}$ with $\operatorname{cr}(Q)=1$. Since $\widetilde{h}_{1}$ and $\widetilde{h}_{2}$ also fix three points $1,-1$ and $i$, it follows from Theorem 4 that $\widetilde{h}_{1}=\widetilde{h}_{2}$. Using Proposition 1. the Beltrami coefficients $\mu_{e x\left(A_{1 n} \circ h_{1} \circ \gamma_{n}\right)}$ and $\mu_{e x\left(A_{2 n} \circ h_{2} \circ \gamma_{n}\right)}$ converge to $\mu_{e x\left(\widetilde{h}_{1}\right)}$ and $\mu_{e x\left(\widetilde{h}_{2}\right)}$ uniformly on $D_{0}$ respectively; that is,

$$
\left.|| \mu_{e x\left(A_{1 n} \circ h_{1} \circ \gamma_{n}\right)}\right|_{D_{0}}-\left.\left.\mu_{e x\left(A_{2 n} \circ h_{2} \circ \gamma_{n}\right)}\right|_{D_{0}}\right|_{L^{\infty}} \rightarrow 0 .
$$

On the other hand, by (2.2) and the conformal naturality of Douady-Earle extensions,

$$
\begin{aligned}
& || \mu_{e x\left(A_{1 n} \circ h_{1} \circ \gamma_{n}\right)}\left|D_{0}-\mu_{e x\left(A_{2 n} \circ h_{2} \circ \gamma_{n}\right)}\right| D_{0}||_{L^{\infty}} \\
= & \|\left.\mu_{e x\left(h_{1}\right)}\left|D_{n}-\mu_{e x\left(h_{2}\right)}\right|_{D_{n}}\right|_{L^{\infty}} \geq \varepsilon_{0} .
\end{aligned}
$$

This is a contradiction to (2.4). Thus, $h_{1} \circ\left(h_{2}\right)^{-1}$ is symmetric and hence $\left[h_{1}\right]=\left[h_{2}\right]$.

In the remaining part of this section, we prove the equivalence of the topologies induced by $d_{A T}, d_{A M}$ and $d_{A C}$ on $A T(\mathbb{D})$ in the same order as we have shown the equivalence of the topologies induced by $d_{T}, d_{M}$ and $d_{C}$ on $T(\mathbb{D})$.

Proof of Theorem \%. Let $[h] \in A T(\mathbb{D})$ and $\left\{\left[h_{n}\right]\right\}_{n=1}^{\infty}$ be a sequence of points in $A T(\mathbb{D})$. 
Characterization of Asymptotic Teichmüller Space Through Shears 527

At first, as pointed out in the introduction, $d_{A M}\left(\left[h_{n}\right],[h]\right) \leq$ $d_{A T}\left(\left[h_{n}\right],[h]\right)$. Thus, $d_{A T}\left(\left[h_{n}\right],[h]\right) \rightarrow 0$ implies $d_{A M}\left(\left[h_{n}\right],[h]\right) \rightarrow 0$ as $n \rightarrow$ $\infty$.

Secondly, we show $d_{A M}\left(\left[h_{n}\right],[h]\right) \rightarrow 0$ implies $d_{A C}\left(\left[h_{n}\right],[h]\right) \rightarrow 0$ as $n \rightarrow \infty$. If on the contrary this is not true, then there exists a subsequence of $\left\{\left[h_{n}\right]\right\}_{n=1}^{\infty}$, denoted again by $\left\{\left[h_{n}\right]\right\}_{n=1}^{\infty}$ for simplicity, such that $d_{A C}\left(\left[h_{n}\right],[h]\right)>\varepsilon_{0}$ for all $n$ and some $\varepsilon_{0}>0$. Then, for each $n$, there is a degenerating sequence $\left\{Q_{n_{j}}=\left\{a_{n_{j}}, b_{n_{j}}, c_{n_{j}}, d_{n_{j}}\right\}\right\}_{j=1}^{\infty}$ of quadruples and $\varepsilon_{0}>0$ such that, for each $n$,

$$
\lim _{j \rightarrow \infty} \frac{c r\left(h_{n}\left(Q_{n_{j}}\right)\right)}{\operatorname{cr}\left(h\left(Q_{n_{j}}\right)\right)}>e^{\varepsilon_{0}} .
$$

Thus, we can choose a degenerating sequence $\left\{Q_{n}=\left\{a_{n}, b_{n}, c_{n}, d_{n}\right\}\right\}_{n=1}^{\infty}$ of quadruples such that for each $n$,

$$
\frac{\operatorname{cr}\left(h_{n}\left(Q_{n}\right)\right)}{\operatorname{cr}\left(h\left(Q_{n}\right)\right)}>e^{\varepsilon_{0}}
$$

Using the same argument in the second step in the proof of Theorem 5, we obtain

$$
d_{\rho_{(0,-1)}}\left(\operatorname{cr}\left(h_{n}\left(Q_{n}\right), \operatorname{cr}\left(h\left(Q_{n}\right)\right)\right)>\varepsilon_{0}^{\prime}\right.
$$

for all $n$ and some $\varepsilon_{0}^{\prime}>0$. By Lemma 1 , for all $n$,

$$
\left|\log \frac{M\left(h_{n}\left(Q_{n}\right)\right)}{M\left(h\left(Q_{n}\right)\right)}\right|>\varepsilon_{0}^{\prime}
$$

which is a contradiction to the assumption that $d_{A M}\left(\left[h_{n}\right],[h]\right) \rightarrow 0$ as $n \rightarrow$ $\infty$.

Finally, we show that $d_{A C}\left(\left[h_{n}\right],[h]\right) \rightarrow 0$ implies $d_{A T}\left(\left[h_{n}\right],[h]\right) \rightarrow 0$. Suppose on the contrary that $d_{A T}\left(\left[h_{n}\right],[h]\right) \nrightarrow 0$. Passing to a subsequence, we may assume that for all $n$,

$$
d_{A T}\left(\left[h_{n}\right],[h]\right)>\varepsilon>0 .
$$

Let $e x\left(h_{n}\right)$ and $e x(h)$ be the Douady-Earle extensions of $h_{n}$ and $h$ respectively. Using (2.5), we can choose a sequence of hyperbolic disks $\left\{D_{n}\right\}_{n=1}^{\infty} \subset$ $\mathbb{D}$ of diameter 1 with the Euclidean distance from $D_{n}$ to $\mathbb{S}^{1}$ approaching 0 
as $n \rightarrow \infty$ such that for all $n$,

$$
\left\|\left.\mu_{e x\left(h_{n}\right)}\right|_{D_{n}}-\mu_{e x(h)} \mid D_{n}\right\|_{L^{\infty}} \geq \epsilon .
$$

Let $D_{0}$ be the hyperbolic disk on $\mathbb{D}$ of diameter 1 and centered at 0 , and assume that $\gamma_{n} \in M \ddot{o} b(\mathbb{D})$ and $\gamma_{n}\left(D_{0}\right)=D_{n}$. Let $A_{1 n}$ and $A_{2 n} \in M \ddot{o} b(\mathbb{D})$ such that $A_{1 n} \circ h_{n} \circ \gamma_{n}$ and $A_{2 n} \circ h \circ \gamma_{n}$ fix $1,-1, i$ for each $n$. Now given a quadruple $Q$ with $\operatorname{cr}(Q)=1$, it follows from the sublemma in the proof of the previous theorem that $s\left(\gamma_{n}(Q)\right) \rightarrow 0$ as $n \rightarrow \infty$. Using the assumption that $d_{A C}\left(\left[h_{n}\right],[h]\right) \rightarrow 0$ as $n \rightarrow \infty$, we obtain

$$
\lim _{n \rightarrow \infty}\left|\frac{c r\left(A_{1 n} \circ h_{n} \circ \gamma_{n}(Q)\right)}{c r\left(A_{2 n} \circ h \circ \gamma_{n}(Q)\right)}\right|=1
$$

Since $d_{A C}\left(\left[h_{n}\right],[h]\right) \rightarrow 0$ as $n \rightarrow \infty$, it follows that $\left\{d_{A C}\left(\left[h_{n}\right],[I d]\right)\right\}_{n=1}^{\infty}$ is bounded. Using the definition of $d_{A C}$ and Lemma 2, we know that $\left\{H\left(e x\left(h_{n}\right)\right)\right\}_{n=1}^{\infty}$ is bounded. For each $n$, we can choose $0<r_{n}<1$ such that $\frac{1+\left\|\mu_{n}\right\|_{\infty}}{1-\left\|\mu_{n}\right\|_{\infty}} \leq H\left(\operatorname{ex}\left(h_{n}\right)\right)+1$, where $\mu_{n}$ is defined as

$$
\mu_{n}(z)=\left\{\begin{array}{lc}
\mu_{e x\left(h_{n}\right)}(z), & r_{n} \leq|z|<1 \\
0, & |z|<r_{n}
\end{array}\right.
$$

Let $f_{n}$ be the normalized (i.e., fixing three points $1,-1$ and $i$ ) quasiconformal homeomorphism of $\mathbb{D}$ with the Beltrami coefficient $\mu_{n}$, and $\widetilde{h}_{n}=\left.f_{n}\right|_{\mathbb{S}^{1}}$. Then $\widetilde{h}_{n} \in\left[h_{n}\right]$ for each $n$ and $\left\{K\left(\operatorname{ex}\left(\widetilde{h}_{n}\right)\right)\right\}_{n=1}^{\infty}$ is bounded. For simplicity, we denote $\left\{\left[\widetilde{h}_{n}\right]\right\}_{n=1}^{\infty}$ by $\left\{\left[h_{n}\right]\right\}_{n=1}^{\infty}$. In other words, by replacing representatives of $\left[h_{n}\right]^{\prime} s$, we may assume that $\left\{K\left(e x\left(h_{n}\right)\right)\right\}_{n=1}^{\infty}$ is bounded.

Since all $A_{1 n} \circ h_{n} \circ \gamma_{n}$ and $A_{2 n} \circ h \circ \gamma_{n}$ are normalized to fix three points, passing to subsequences we may assume that $A_{1 n} \circ h_{n} \circ \gamma_{n}$ and $A_{2 n} \circ h \circ \gamma_{n}$ converge uniformly to quasisymmetric maps $h^{*}$ and $\widehat{h}^{*}$ on $\mathbb{S}^{1}$ respectively. Using (2.7) and the convergence of the two sequences, we obtain

$$
\operatorname{cr}\left(h^{*}(Q)\right)=\operatorname{cr}\left(\widehat{h}^{*}(Q)\right)
$$

for any quadruple $Q$ with $\operatorname{cr}(Q)=1$. Thus, $h^{*}=\widehat{h}^{*}$. Using Proposition 1, we obtain

$$
\left.|| \mu_{e x\left(A_{1 n} \circ h_{n} \circ \gamma_{n}\right)}\left|D_{0}-\mu_{e x\left(A_{2 n} \circ h \circ \gamma_{n}\right)}\right|_{D_{0}}\right|_{L^{\infty}} \rightarrow 0 .
$$


On the other hand, (2.6) and the conformal naturality of Douady-Earle extensions imply that for each $n$,

$$
\begin{aligned}
& \|\left.\mu_{e x\left(A_{1 n} \circ h_{n} \circ \gamma_{n}\right)}\left|D_{0}-\mu_{e x\left(A_{2 n} \circ h \circ \gamma_{n}\right)}\right| D_{0}\right|_{L^{\infty}} \\
= & \left\|\mu_{e x\left(h_{n}\right)}\left|D_{n}-\mu_{e x(h)}\right| D_{n}\right\|_{L^{\infty}} \geq \epsilon .
\end{aligned}
$$

This is a contradiction to (2.8). Thus, $d_{A C}\left(\left[h_{n}\right],[h]\right) \rightarrow 0$ implies $d_{A T}\left(\left[h_{n}\right],[h]\right) \rightarrow 0$ as $n \rightarrow \infty$.

\section{A counter-example and a new lemma}

In this section, we construct a sequence $\left\{h_{n}\right\}_{n=2}^{\infty}$ of orientation-preserving homeomorphisms of $\mathbb{S}^{1}$ such that for each $n \geq 2, h_{n}$ fixes four points \pm 1 and $\pm i$ and the Douady-Earle extension $e x\left(h_{n}\right)$ of $h_{n}$ fixes the origin, but the maximal dilatation $K\left(e x\left(h_{n}\right)\right)(0)$ of $e x\left(h_{n}\right)$ at the origin goes to $\infty$ as $n \rightarrow \infty$. Let $A$ be the Möbius transformation from the unit disk $\mathbb{D}$ to the upper half plane $\mathbb{H}$ mapping $-1,-i$ and 1 to $-1,0$ and 1 respectively. Then the sequence $\left\{A \circ h_{n} \circ A^{-1}\right\}_{n=2}^{\infty}$ is a counter-example to Lemma 2.2 in [21] (the statement of this lemma is recalled in the introduction).

Given each $n \geq 2$, we first define the map $h_{n}$ on the circular arc of $\mathbb{S}^{1}$ in the first quadrant; that is, $h_{n}$ maps the circular arc from 1 to $e^{\frac{\pi}{2}\left(1-\frac{1}{n}\right) i}$ linearly onto the arc from 1 to $e^{\frac{\pi}{2 n} i}$ and maps the arc from $e^{\frac{\pi}{2}\left(1-\frac{1}{n}\right) i}$ to $i$ linearly onto the arc from $e^{\frac{\pi}{2 n} i}$ to $i$. Clearly, both 1 and $i$ are fixed under $h_{n}$ and $e^{\frac{\pi}{2}\left(1-\frac{1}{n}\right) i}$ is mapped to $e^{\frac{\pi}{2 n} i}$. Through conjugation under taking conjugates of complex numbers, $h_{n}$ is extended to be defined on the arc of $\mathbb{S}^{1}$ in the fourth quadrant. Finally, through conjugation under taking mirror images with respect to the imaginary axis, $h_{n}$ is extended to be defined on $\mathbb{S}^{1}$. Note that $h_{2}$ is equal to the identity map on $\mathbb{S}^{1}$. Since each $h_{n}, n \geq 2$, is symmetric with respect to the origin, using definition we see that the Douady-Earle extension $\operatorname{ex}\left(h_{n}\right)$ fixes the origin. The main work is to show that

$$
\left|\mu_{e x\left(h_{n}\right)}(0)\right| \rightarrow 1 \text { as } n \rightarrow \infty .
$$

Before starting to show this convergence, let us first recall some formulas to express the Beltrami coefficient of the Douady-Earle extension at the origin.

Let $h$ be an orientation-preserving homeomorphism of $\mathbb{S}^{1}$. Assume that the Douady-Earle extension $e x(h)$ is normalized at the origin; that is, $e x(h)(0)=0$. Then the Beltrami coefficient of $e x(h)$ at the origin can be 
explicitly expressed as follows [2]. Given any point $z \in \mathbb{D}, e x(h)(z)$ is equal to the unique point $w \in \mathbb{D}$ such that

$$
F(z, w)=0
$$

where

$$
F(z, w)=\frac{1}{2 \pi} \int_{\mathbb{S}^{1}} \frac{h(\xi)-w}{1-\bar{w} h(\xi)} \cdot \frac{1-|z|^{2}}{|z-\xi|^{2}}|d \xi|
$$

If we let

$$
c_{1}=\frac{\partial F}{\partial z}(0,0)=\frac{1}{2 \pi} \int_{\mathbb{S}^{1}} \bar{\xi} h(\xi)|d \xi|, \quad c_{-1}=\frac{\partial F}{\partial \bar{z}}(0,0)=\frac{1}{2 \pi} \int_{\mathbb{S}^{1}} \xi h(\xi)|d \xi|
$$

and

$$
d_{1}=\frac{\partial F}{\partial w}(0,0)=-1, \quad d_{-1}=\frac{\partial F}{\partial \bar{w}}(0,0)=\frac{1}{2 \pi} \int_{\mathbb{S}^{1}} h(\xi)^{2}|d \xi|
$$

then

$$
\frac{\partial w}{\partial \bar{z}}(0)=-\frac{\overline{\frac{\partial F}{\partial w}(0,0)} \frac{\partial F}{\partial \bar{z}}(0,0)-\frac{\partial F}{\partial \bar{w}}(0,0) \frac{\overline{\partial F}(0,0)}{\partial z}}{\left|\frac{\partial F}{\partial w}(0,0)\right|^{2}-\left|\frac{\partial F}{\partial \bar{w}}(0,0)\right|^{2}}=\frac{c_{-1}+d_{-1} \bar{c}_{1}}{1-\left|d_{-1}\right|^{2}}
$$

and

$$
\frac{\partial w}{\partial z}(0)=-\frac{\overline{\frac{\partial F}{\partial w}(0,0)} \frac{\partial F}{\partial z}(0,0)-\frac{\partial F}{\partial \bar{w}}(0,0) \frac{\overline{\partial F}(0,0)}{\partial \bar{z}}(0,0)}{\left|\frac{\partial F}{\partial w}(0,0)\right|^{2}-\left|\frac{\partial F}{\partial \bar{w}}(0,0)\right|^{2}}=\frac{c_{1}+d_{-1} \bar{c}_{-1}}{1-\left|d_{-1}\right|^{2}}
$$

Therefore the Beltrami coefficient $\mu_{e x(h)}$ of $e x(h)$ at 0 is equal to

$$
\mu_{e x(h)}(0)=\frac{c_{-1}+d_{-1} \bar{c}_{1}}{c_{1}+d_{-1} \bar{c}_{-1}}
$$

If the boundary homeomorphism $h$ satisfies $h(\bar{\xi})=\overline{h(\xi)}$ and $h(-\bar{\xi})=$ $-\overline{h(\xi)}$, then one can rewrite

$$
c_{1}=\frac{2}{\pi} \operatorname{Re} \int_{0}^{\frac{\pi}{2}} \bar{\xi} h(\xi)|d \xi|, \quad c_{-1}=\frac{2}{\pi} \operatorname{Re} \int_{0}^{\frac{\pi}{2}} \xi h(\xi)|d \xi|
$$

and

$$
d_{-1}=\frac{2}{\pi} R e \int_{0}^{\frac{\pi}{2}} h^{2}(\xi)|d \xi| .
$$


Characterization of Asymptotic Teichmüller Space Through Shears 531

Now let $h=h_{n}$. In the following, we show that both $c_{1}$ and $c_{-1}$ converge to $\frac{2}{\pi}$ and $d_{-1}$ converges to 1 as $n \rightarrow \infty$. It follows that $\mu_{e x\left(h_{n}\right)}(0)$ converges to 1 as $n \rightarrow \infty$.

We first show that $c_{1}$ converges to $\frac{2}{\pi}$ as $n \rightarrow \infty$.

Let $\theta_{n}=\frac{\pi}{2 n}$, where $n \geq 2$. Then for each $\xi=e^{i \theta}$ with $0 \leq \theta \leq \frac{\pi}{2}-\theta_{n}$, $h(\xi)=1+O\left(\frac{1}{n}\right)$. Clearly,

$$
\int_{0}^{\frac{\pi}{2}} \bar{\xi} h(\xi)|d \xi|=\int_{0}^{\frac{\pi}{2}-\theta_{n}} \bar{\xi} h(\xi)|d \xi|+\int_{\frac{\pi}{2}-\theta_{n}}^{\frac{\pi}{2}} \bar{\xi} h(\xi)|d \xi| .
$$

Then

$$
\begin{aligned}
\int_{0}^{\frac{\pi}{2}-\theta_{n}} \bar{\xi} h(\xi)|d \xi| & =\int_{0}^{\frac{\pi}{2}-\theta_{n}} \bar{\xi}\left(1+O\left(\frac{1}{n}\right)\right)|d \xi|=\int_{0}^{\frac{\pi}{2}-\theta_{n}} \bar{\xi}|d \xi|+O\left(\frac{1}{n}\right) \\
& =\int_{0}^{\frac{\pi}{2}} \bar{\xi}|d \xi|+O\left(\frac{1}{n}\right)=1-i+O\left(\frac{1}{n}\right)
\end{aligned}
$$

and

$$
\left|\int_{\frac{\pi}{2}-\theta_{n}}^{\frac{\pi}{2}} \bar{\xi} h(\xi)\right| d \xi|| \leq \int_{\frac{\pi}{2}-\theta_{n}}^{\frac{\pi}{2}}|\bar{\xi} h(\xi)||d \xi|=\theta_{n}=O\left(\frac{1}{n}\right) .
$$

Thus

$$
\int_{0}^{\frac{\pi}{2}} \bar{\xi} h(\xi)|d \xi|=1-i+O\left(\frac{1}{n}\right)
$$

and then

$$
c_{1}=\frac{2}{\pi}+O\left(\frac{1}{n}\right) .
$$

It follows that $c_{1}$ converges to $\frac{2}{\pi}$ as $n \rightarrow \infty$.

Similarly, one can show that $c_{-1}$ converges to $\frac{2}{\pi}$ as $n \rightarrow \infty$.

It remains to show that $d_{-1}$ converges to 1 as $n \rightarrow \infty$. Since for each $\xi=$ $e^{i \theta}$ with $0 \leq \theta \leq \frac{\pi}{2}-\theta_{n}, h(\xi)=1+O\left(\frac{1}{n}\right)$ implies $h^{2}(\xi)=1+O\left(\frac{1}{n}\right)$. Then

$$
\begin{aligned}
d_{-1} & =\frac{2}{\pi} \operatorname{Re}\left(\int_{0}^{\frac{\pi}{2}-\theta_{n}} h^{2}(\xi)|d \xi|+\int_{\frac{\pi}{2}-\theta_{n}}^{\frac{\pi}{2}} h^{2}(\xi)|d \xi|\right) \\
& =\frac{2}{\pi} \operatorname{Re}\left(\int_{0}^{\frac{\pi}{2}-\theta_{n}}\left(1+O\left(\frac{1}{n}\right)\right)|d \xi|+O\left(\frac{1}{n}\right)\right) \\
& =\frac{2}{\pi} \operatorname{Re}\left(\frac{\pi}{2}+O\left(\frac{1}{n}\right)\right)=1+O\left(\frac{1}{n}\right) .
\end{aligned}
$$

Thus, $d_{-1}$ converges to 1 as $n \rightarrow \infty$. The proof of (3.1) is complete. 
In the second half of this section, we introduce a new lemma which is used in the next section to prove Theorem 3. This bridges the gap in the proof of Theorem A in [21].

Lemma 3. Let $M>0$ and $h$ be an orientation-preserving homeomorphism of $\mathbb{S}^{1}$. Assume that $p_{1}=\frac{3+4 i}{5}, p_{2}=-\bar{p}_{1}, p_{3}=-p_{1}$ and $p_{4}=\bar{p}_{1}$. If $|\log \operatorname{cr}(h(Q))|<M$ holds for any of the following five quadruples

$$
\begin{gathered}
Q_{0}=\{-1,-i, 1, i\}, Q_{1}=\left\{-1,1, p_{1}, i\right\}, Q_{2}=\left\{-1,1, i, p_{2}\right\}, \\
Q_{3}=\left\{-1, p_{3},-i, 1\right\} \text { and } Q_{4}=\left\{-1,-i, p_{4}, 1\right\},
\end{gathered}
$$

then there exist a universal small neighborhood $U$ of the origin and a constant $C>1$, only depending on $U$ and $M$, such that

$$
K\left(\left.\operatorname{ex}(h)\right|_{U}\right)<C .
$$

Proof. Using the conformal naturality of Douady-Earle extensions and invariance of the cross-ratio distortion norm of $h$ under postcomposition by Möbius transformations, we can assume that the Douady-Earle extension $e x(h)$ fixes the origin. Using Lemma 3.6 in [17, the conclusion of this lemma follows from the following claim.

Claim. There exist $0<s_{0}<\pi$ (universal, one may take $s_{0}=\frac{2 \pi}{3}$ ) and $\epsilon>0$ (only depending on $M$ ) such that for any circular arc $\Gamma$ of length $\geq s_{0}$ and $\leq \pi$, the length of $h(\Gamma)$ is greater than $\epsilon$.

We divide the proof of this claim into the following four steps.

Step 1. In this step, we show there exists a constant $\epsilon_{1}>0$ only depending on $M$ such that for each half circle $\Gamma$ bounded by two of the four points $\{ \pm 1, \pm i\}$, the length $|h(\Gamma)|$ of $h(\Gamma)$ is greater than $\epsilon_{1}$. For example, we show in detail how the two conditions ex $(h)(0)=0$ and $\left|\log \operatorname{cr}\left(h\left(Q_{2}\right)\right)\right|<M$ imply that the length $|h(\widehat{-i, 1, i})|$ is greater than $\epsilon_{1}$, where $\widehat{-i, 1, i}$ denotes the right half circle.

Let $A=-1, B=-i, C=1$ and $D=i$, and let $A^{\prime}=h(A), B^{\prime}=h(B)$, $C^{\prime}=h(C), D^{\prime}=h(D)$ and $p_{k}^{\prime}=h\left(p_{k}\right)$, where $k=1,2,3,4$.

Using the normalization condition $e x(h)(0)=0$, it is shown in Step 1 of the proof of Lemma 1 in [11] that

$$
\left|\widehat{A^{\prime}} \widehat{B^{\prime} C^{\prime}} D^{\prime}\right| \geq \frac{\pi}{3}
$$

where $\widehat{A^{\prime} \widehat{B^{\prime} C^{\prime}} D^{\prime}}$ represents the image under $h$ of the three quarter of the circle from -1 to $i$ and through $-i$ and 1 in the counterclockwise direction. 
Furthermore, it is shown in Case 1 in Step 2 of the proof of Lemma 1 in [11] that

$$
\left|\widehat{B^{\prime} C^{\prime} D^{\prime}} p_{2}^{\prime}\right|>\frac{\pi}{3}
$$

where $\widehat{B^{\prime} C^{\prime} D^{\prime}} p_{2}^{\prime}$ represents the image under $h$ of the circular arc from $-i$ to $p_{2}$ and through 1 and $i$ in the counterclockwise direction.

Now we apply the condition $\left|\log \operatorname{cr}\left(h\left(Q_{2}\right)\right)\right|<M$ to show that there exists $\epsilon_{1}>0$ such that

$$
\left|\widehat{B^{\prime} C^{\prime} D^{\prime}}\right|>\epsilon_{1} .
$$

Let $l=\left|\widehat{B^{\prime} C^{\prime} D^{\prime}}\right|$. Then $l_{1}=\left|\widehat{C^{\prime} p_{1}^{\prime} D^{\prime}}\right|<l$ since $\widehat{C^{\prime} p_{1}^{\prime} D^{\prime}} \subsetneq \widehat{B^{\prime} C^{\prime} D^{\prime}}$. It follows that

$$
\left|\widehat{D^{\prime} p_{2}^{\prime}}\right|=\left|\widehat{B^{\prime} C^{\prime} D^{\prime}} p_{1}^{\prime}\right|-\left|\widehat{B^{\prime} C^{\prime} D^{\prime}}\right|>\frac{\pi}{3}-l
$$

and

$$
\left|\widehat{A^{\prime} B^{\prime} C^{\prime}}\right|=\left|\widehat{A^{\prime} B^{\prime} C^{\prime}} D^{\prime}\right|-\left|\widehat{C^{\prime} p_{1}^{\prime} D^{\prime}}\right|>\frac{2 \pi}{3}-l_{1}>\frac{2 \pi}{3}-l .
$$

Then

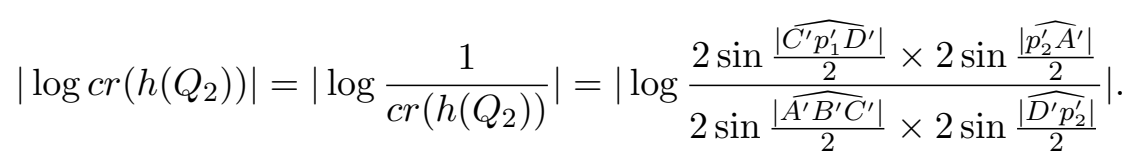

Clearly,

$$
\frac{2 \sin \frac{\left|\widehat{C^{\prime} p_{1}^{\prime} D^{\prime}}\right|}{2} \times 2 \sin \frac{\left|\widehat{p_{2}^{\prime} A^{\prime}}\right|}{2}}{2 \sin \frac{\left|\widehat{A^{\prime} B^{\prime} C^{\prime}}\right|}{2} \times 2 \sin \frac{\left|\widehat{D^{\prime} p_{2}^{\prime}}\right|}{2}}<\frac{2 \sin \frac{l}{2} \times 2}{2 \sin \left(\frac{\pi}{3}-\frac{l}{2}\right) \times 2 \sin \left(\frac{\pi}{6}-\frac{l}{2}\right)} .
$$

Thus this quotient approaches 0 as $l \rightarrow 0$, which implies that $\left|\log \operatorname{cr}\left(h\left(Q_{2}\right)\right)\right|$ goes to $\infty$ as $l \rightarrow 0$. This is a contradiction to $\left|\log \operatorname{cr}\left(h\left(Q_{2}\right)\right)\right|<M$. Therefore, there exists $\epsilon_{1}>0$ depending on $M$ such that $\left|\widehat{B^{\prime} C^{\prime} D^{\prime}}\right|>\epsilon_{1}$.

Similarly, using $\operatorname{ex}(h)(0)=0$ and $\left|\log \operatorname{cr}\left(h\left(Q_{3}\right)\right)\right|<M$, we show $\left|\widehat{C^{\prime} D^{\prime} A^{\prime}}\right|>\epsilon_{1} ; \quad$ using $\operatorname{ex}(h)(0)=0$ and $\left|\log \operatorname{cr}\left(h\left(Q_{4}\right)\right)\right|<M$, we show $\left|\widehat{D^{\prime} A^{\prime} B^{\prime}}\right|>\epsilon_{1} ; \quad$ using $\operatorname{ex}(h)(0)=0$ and $\left|\log \operatorname{cr}\left(h\left(Q_{1}\right)\right)\right|<M$, we show $\left|\widehat{A^{\prime} B^{\prime} C^{\prime}}\right|>\epsilon_{1}$.

Step 2. Using the estimates on the lengths of the images of the four half circles obtained in Step 1 and the condition that $\left|\log \operatorname{cr}\left(h\left(Q_{0}\right)\right)\right|<M$, we can apply the strategy to derive the existence of $\epsilon_{1}$ to show that there exists $\epsilon_{2}>0$ such that the length of the image, under $h$, of each of the four quarters $\widehat{C p_{1} D}, \widehat{D p_{2} A}, \widehat{A p_{3} B}$ and $\widehat{B p_{4} C}$ of the circle is greater than $\epsilon_{2}$. 
Step 3. Using the estimates on the lengths of the images of the four half circles obtained in Step 1, the estimates on the lengths of the images of the four quarters obtained in Step 2, and the condition that $\left|\log \operatorname{cr}\left(h\left(Q_{k}\right)\right)\right|<M$ for $k=1,2,3,4$, we can apply the strategy to derive the existence of $\epsilon_{1}$ to show that there exists $\epsilon_{3}>0$ such that the length of the image, under $h$, of each of the eight circular arcs bounded by adjacent points of $\left\{-1, p_{3},-i, p_{4}, 1, p_{1}, i, p_{2}\right\}$ on $\mathbb{S}^{1}$ is greater than $\epsilon_{3}$.

Step 4. Let $s_{0}=\frac{2 \pi}{3}$ and $\epsilon=\epsilon_{3}$. Then for any circular arc $\Gamma$ with length $s_{0} \leq|\Gamma| \leq \pi, \Gamma$ contains at least one circular arc between two adjacent points of $\left\{-1, p_{3},-i, p_{4}, 1, p_{1}, i, p_{2}\right\}$ on $\mathbb{S}^{1}$ since two longest such arcs (among the eight such arcs) comprise an arc of length $2 \arcsin \frac{4}{5}<\frac{2 \pi}{3}$. Therefore, the length $|h(\Gamma)|>\epsilon_{3}=\epsilon$.

\section{The Farey tessellation, shear maps, and proof of Theorem}

3

We first introduce some background on the Farey tessellation and shear maps. Then we prove Theorem 3 .

In the following, we use the upper half plane $\mathbb{H}$ as a model for the hyperbolic plane and recall the Farey tessellation and the shear map, which were introduced by Penner in [19] and further studied by Šarić in [21] and [22].

Let $\triangle_{0}$ be the ideal geodesic triangle on $\mathbb{H}$ with vertices 0,1 and $\infty$ and let $\Gamma$ be the group generated by the hyperbolic reflections to the sides of $\triangle_{0}$. The Farey tessellation $\mathcal{F}$ is the collection of the geodesics on the $\Gamma$-orbits of the edges of $\triangle_{0}$. The set of the endpoints of the geodesics in $\mathcal{F}$ is equal to $\widehat{\mathbb{Q}}=\mathbb{Q} \cup\{\infty\}$.

Let $e \in \mathcal{F}$ and let $a, c \in \widehat{\mathbb{R}}$ be the endpoints of $e$. Assume that $\left(\triangle_{1}, \triangle_{2}\right)$ is a pair of two triangles on $\mathbb{H}$ with disjoint interiors and sharing a common boundary $e$. Besides the vertices $a$ and $c$, we denote the third vertices of $\triangle_{1}$ and $\triangle_{2}$ by $b$ and $d$ such that $a, b, c$ and $d$ are arranged in the counterclockwise order on $\widehat{\mathbb{R}}$. Given a homeomorphism $h: \widehat{\mathbb{R}} \rightarrow \widehat{\mathbb{R}}$, the shear $s_{h}(e)$ mentioned in the introduction can be precisely defined by

$$
s_{h}(e)=\log \operatorname{cr}(h(\{a, b, c, d\})),
$$

where $\operatorname{cr}(h(\{a, b, c, d\}))$ is the cross ratio defined by $(1.2)$ in the introduction. Then $h: \widehat{\mathbb{R}} \rightarrow \widehat{\mathbb{R}}$ induces a map $s_{h}: \mathcal{F} \rightarrow \mathbb{R}$, which is called the shear map or function or coordinate of $h$. Conversely, given any map $s: \mathcal{F} \rightarrow \mathbb{R}$, there 
Characterization of Asymptotic Teichmüller Space Through Shears 535

is a unique injective map $h_{s}$ from the vertices $\widehat{\mathbb{Q}}$ of the Farey tessellation $\mathcal{F}$ into $\widehat{\mathbb{R}}$ such that $h_{s}$ fixes 0,1 and $\infty$ and the shear map of $h_{s}$ is equal to $s$. We call $h_{s}$ the characteristic map of $s$.

A fan $\mathcal{F}_{p}$ of geodesics in $\mathcal{F}$ with tip $p \in \widehat{\mathbb{Q}}$ consists of all edges of $\mathcal{F}$ that have a common endpoint at $p$. Each fan $\mathcal{F}_{p}$ has a natural order. Take a horocycle $C$ tangent to $\widehat{\mathbb{R}}$ at $p$ and choose an orientation on $C$ such that the corresponding horoball is to the left of $C$. Let $e, e^{\prime}$ be two geodesics in $\mathcal{F}_{p}$. Define $e<e^{\prime}$ if the point $e \cap C$ comes before the point $e^{\prime} \cap C$. This natural order on $\mathcal{F}_{p}$ gives a one-to-one correspondence between $\mathcal{F}_{p}$ and the set $\mathbb{Z}$ of integers, and any two such correspondences differ by a translation in $\mathbb{Z}$.

Given a shear map $s$ and a fan $\mathcal{F}_{p}=\left\{e_{n}^{p}\right\}_{n \in \mathbb{Z}}$, the quantity $s(p ; m, k)$ is defined by (1.1) in the introduction.

Remark 1. Let $s: \mathcal{F} \rightarrow \mathbb{R}$ be a map from $\mathcal{F}$ to $\mathbb{R}$ and let $h_{s}$ be the characteristic map of $s$. Assume that $e_{j}^{p}, j=m-k, m, m+k$, are three geodesics in a fan $\mathcal{F}_{p}$. Let $a_{j}^{p}$ be the other endpoint of $e_{j}^{p}$ besides $p$, where $j=m-k, m, m+k$. Using the definition of cross ratio given by (1.2) and through pre-composition by a Möbius transformation to arrange $p$ at $\infty$, one can easily see that for any $m, k \in \mathbb{Z}$,

$$
\operatorname{cr}\left(\left\{p, a_{m-k}^{p}, a_{m}^{p}, a_{m+k}^{p}\right\}\right)=1 .
$$

Similarly, through post-composition by a Möbius transformation to arrange $h_{s}(p)$ at $\infty$, we see

$$
s(p ; m, k)=\operatorname{cr}\left(h_{s}\left(\left\{p, a_{m-k}^{p}, a_{m}^{p}, a_{m+k}^{p}\right\}\right)\right) .
$$

Using the definitions of $d_{S}$ and $d_{C}$ in the introduction, we know that for any $h_{1}, h_{2} \in T(\mathbb{D})$,

$$
d_{S}\left(s_{1}, s_{2}\right) \leq d_{C}\left(h_{1}, h_{2}\right),
$$

where $s_{1}$ and $s_{2}$ are the shear functions induced by $h_{1}$ and $h_{2}$.

Substituting $h_{2}$ by the identity map on $\mathbb{S}^{1}$ in the previous inequality, one can see that the shear norm $M_{s_{1}}$ of $h_{1}$ is finite is a necessary condition for $s_{1}$ to be induced by a quasisymmetric map. This verifies the necessity of the condition in Theorem $A$ of [21] for a shear function to be induced by a quasisymmetric homeomorphism.

Using the previous inequality and Theorem 5, we can conclude that as $n \rightarrow \infty$,

$$
d_{T}\left(h_{n}, h\right) \rightarrow 0 \text { implies } d_{S}\left(s_{n}, s\right) \rightarrow 0 .
$$


It is shown in Theorem $B$ of [21] that as $n \rightarrow \infty$,

$$
d_{T}\left(h_{n}, h\right) \rightarrow 0 \text { if and only if } d_{S}\left(s_{n}, s\right) \rightarrow 0 .
$$

Let $A=-\frac{z-i}{z+i}$ be the Möbius transformation form $\mathbb{H}$ to $\mathbb{D}$ that maps 0,1 and $\infty$ to $1, i$ and -1 respectively. In the following, given a homeomorphism $h: \mathbb{S}^{1} \rightarrow \mathbb{S}^{1}$ of $\mathbb{S}^{1}$, we denote by $\widetilde{h}=A^{-1} \circ h \circ A$, which is a homeomorphism of $\widehat{\mathbb{R}}$ fixing 0,1 and $\infty$. Then Lemma 3 converts to the following corollary.

Corollary 1. Let $M>0$ and $\widetilde{h}$ be a homeomorphism of $\widehat{\mathbb{R}}$ with 0,1 and $\infty$ fixed. If $|\log \operatorname{cr}(\widetilde{h}(Q))|<M$ holds for any of the following five quadruples

$$
\begin{gathered}
Q_{0}=\{\infty,-1,0,1\}, Q_{1}=\left\{\infty, 0, \frac{1}{2}, 1\right\}, Q_{2}=\{\infty, 0,1,2\}, \\
Q_{3}=\{\infty,-2,-1,0\} \text { and } Q_{4}=\left\{\infty,-1,-\frac{1}{2}, 0\right\},
\end{gathered}
$$

then there exist a constant $C>1$ and a neighborhood $U$ of $i$, only depending on $M$, such that

$$
K\left(\left.\operatorname{ex}(\widetilde{h})\right|_{U}\right)<C
$$

Proof of Theorem 3 . Given a homeomorphism $\tilde{h}_{n}$ of $\widehat{\mathbb{R}}$, we denote by $s_{n}$ the shear map of $h_{n}$. Suppose that Theorem 3 fails. Then there exists a sequence $\left\{\widetilde{h}_{n}\right\}_{n=1}^{\infty}$ of homeomorphisms of $\widehat{\mathbb{R}}$ such that $M_{s_{n}}<M$ for all $n$ and $K\left(\operatorname{ex}\left(\widetilde{h}_{n}\right)\right) \rightarrow \infty$ as $n \rightarrow \infty$. Then there exists a sequence $\left\{z_{n}\right\}_{n=1}^{\infty}$ of points on $\mathbb{H}$ such that $\left|\mu_{e x\left(\widetilde{h}_{n}\right)}\left(z_{n}\right)\right| \rightarrow 1$ as $n \rightarrow \infty$. Denote by $\triangle_{n}$ the ideal triangle in $\mathcal{F}$ such that $z_{n} \in \bar{\triangle}_{n}$. Let $A_{n} \in P S L(2, \mathbb{Z})$ such that $A_{n}\left(\triangle_{n}\right)=\triangle_{0}$, and let $B_{n} \in P S L(2, \mathbb{R})$ such that $\widehat{h}_{n}=B_{n} \circ \widetilde{h}_{n} \circ\left(A_{n}\right)^{-1}$ fixes 0,1 and $\infty$.

Let $z_{n}^{\prime}=A_{n}\left(z_{n}\right)$. Consider the following two cases of $\left\{z_{n}^{\prime}\right\}_{n=1}^{\infty}$. One is that a subsequence of $\left\{z_{n}^{\prime}\right\}_{n=1}^{\infty}$ stays in a compact subset of $\bar{\triangle}_{0}$, the other is that $\left\{z_{n}\right\}_{n=1}^{\infty}$ has a subsequence converging to an endpoint of $\triangle_{0}$ as $n \rightarrow \infty$. In the second case, without loss of generality we may assume that $z_{n}$ converges to $\infty$ as $n \rightarrow \infty$.

In the first case, we briefly denote the subsequence by $\left\{z_{n}^{\prime}\right\}_{n=1}^{\infty}$ again. Since $M_{s_{n}}<M$ for all $n$, the sequence of the shear maps $s_{n} \circ A_{n}^{-1}$ corresponding to the homeomorphisms $\widehat{h}_{n}=B_{n} \circ \widetilde{h}_{n} \circ\left(A_{n}\right)^{-1}$ has a convergent subsequence in the sense that for any $e \in \mathcal{F}$, the sequence of real number $s_{n_{k}} \circ A_{n_{k}}^{-1}(e)$ converges as $k \rightarrow \infty$. It is clear that the limiting shear map $s_{\infty}: \mathcal{F} \rightarrow \mathbb{R}$ satisfies $M_{s_{\infty}}<M$. Since all maps $\widehat{h}_{n_{k}}$ are normalized to fix three points, it is shown on pages 2418-9 in [21] that the convergent subsequence $\left\{\widehat{h}_{n_{k}}\right\}_{k=1}^{\infty}$ converges pointwisely to a homeomorphism $\widehat{h}_{\infty}$ 
inducing the shear map $s_{\infty}$. Then $\left|\mu_{e x\left(\widehat{h}_{n_{k}}\right)}\right|$ converges to $\left|\mu_{e x\left(\widehat{h}_{\infty}\right)}\right|$ uniformly on any compact subset of $\mathbb{H}$. Since $\operatorname{ex}\left(\widehat{h}_{\infty}\right)$ is a real analytic diffeomorphism, it follows that for any compact $\Omega$ of $\mathbb{H}$, there exists $0<a<1$ such that $\left|\mu_{e x\left(\widehat{h}_{\infty}\right)}\right|<a$ on $\Omega$. On the other hand, by the conformal naturality of Douady-Earle extensions,

$$
\left|\mu_{e x\left(\widehat{h}_{n_{k}}\right)}\left(z_{n_{k}}^{\prime}\right)\right|=\left|\mu_{e x\left(\widetilde{h}_{n_{k}}\right)}\left(z_{n_{k}}\right)\right| \rightarrow 1 \text { as } k \rightarrow \infty .
$$

This is a contradiction.

Now we show that there will be a contradiction coming up in the second case too. Let $\lambda_{n}$ be the greatest even integer less than or equal to $\operatorname{Im}\left(z_{n}^{\prime}\right)$ and let $\lambda_{n}^{\prime}$ be the real number such that $\widehat{h}_{n}^{*}(x)=\lambda_{n}^{\prime} \widehat{h}_{n}\left(\lambda_{n} x\right)$ fixes 0,1 and $\infty$. We show that $\widehat{h}_{n}^{*}$ satisfies the conditions in Corollary 1 for all $n$. For example, we verify that if $Q=\{\infty,-1,0,1\}$, then for all $n$,

$$
\left|\log \operatorname{cr}\left(\widehat{h}_{n}^{*}(Q)\right)\right| \leq M
$$

Verifications of the above inequality for the other four cases of $Q$ in Corollary 1 are similar. Therefore, we skip them here.

Let $e_{k}^{\infty}$ be the edge in $\mathcal{F}$ that connects an integer $k$ to $\infty$. Using the fan $\left\{e_{k}^{\infty}\right\}_{k \in \mathbb{Z}}$, we obtain

$$
\begin{aligned}
\left|\log \operatorname{cr}\left(\widehat{h}_{n}^{*}(\{\infty,-1,0,1\})\right)\right| & =\left|\log \operatorname{cr}\left(\widehat{h}_{n}\left(\left\{\infty,-\lambda_{n}, 0, \lambda_{n}\right\}\right)\right)\right| \\
& =\left|\log \operatorname{cr}\left(\widetilde{h}_{n} \circ A_{n}^{-1}\left(\left\{\infty,-\lambda_{n}, 0, \lambda_{n}\right\}\right)\right)\right| \\
& \leq M_{s_{n}} \leq M .
\end{aligned}
$$

Let $w_{n}=\left(1 / \lambda_{n}\right) z_{n}^{\prime}$. Then $w_{n}$ converges to the point $i$ as $n \rightarrow \infty$. Therefore,

$$
\left|\mu_{\text {ex }\left(\widehat{h}_{n}^{*}\right)}\left(w_{n}\right)\right|=\left|\mu_{\text {ex }\left(\widehat{h}_{n}\right)}\left(z_{n}^{\prime}\right)\right|=\left|\mu_{e x\left(\widetilde{h}_{n}\right)}\left(z_{n}\right)\right| \rightarrow 1
$$

as $n \rightarrow \infty$. This is a contradiction to Corollary 1 .

The proof is complete.

\section{A metric characterization of $A T(\mathbb{D})$ through equivalence classes of shear maps}

For each edge $e$ in $\mathcal{F}$, we define the Farey generation $g(e)$ of $e$ as follows. Each boundary edge of $\triangle_{0}$ has Farey generation 0 . If an edge $e \in \mathcal{F}$ is obtained by $n$ reflections of an edge of $\triangle_{0}$ (where $n$ is the smallest such number), then the Farey generation $g(e)$ of $e$ is defined to be $n$. 
Given two points $h_{1}$ and $h_{2}$ of $T(\mathbb{D})$, let $s_{1}$ and $s_{2}$ be the shear maps induced by $h_{1}$ and $h_{2}$ respectively. For each fan $\mathcal{F}_{p}=\left\{e_{n}^{p}\right\}_{n \in \mathbb{Z}}$, we let

$$
A M_{s_{1}, s_{2}}(p)=\limsup _{\min \left\{g\left(e_{m+k}^{p}\right), g\left(e_{m-k}^{p}\right)\right\} \rightarrow \infty}\left|\log \frac{s_{1}(p ; m, k)}{s_{2}(p ; m, k)}\right|,
$$

and

$$
d_{A S}\left(s_{1}, s_{2}\right)=\sup _{p \in \mathcal{P}} A M_{s_{1}, s_{2}}(p),
$$

where $\mathcal{P}$ is the collection of all endpoints of the geodesics in $\mathcal{F}$.

Recall that $\mathcal{X}$ is the collection of all real-valued functions $s: \mathcal{F} \rightarrow \mathbb{R}$ with finite shear norms, and $\mathcal{X}$ gives a parametrization of $T(\mathbb{D})$. Then it is clear that $d_{A S}$ defines a pseudo metric on $\mathcal{X}$ and hence introduces an equivalence relation on $\mathcal{X}$ by defining two elements $s$ and $s^{\prime}$ of $\mathcal{X}$ to be equivalent if $d_{A S}\left(s, s^{\prime}\right)=0$. We denote by $[s]$ the equivalence class of $s$ and by $\mathcal{A X}$ the quotient space under this equivalent relation. The pseudo metric $d_{A S}$ on $\mathcal{X}$ induces a metric on $\mathcal{A X}$, which is called the asymptotic shear metric on $\mathcal{A X}$ and still denoted by $d_{A S}$. In this section, we prove our Theorems 1 and 2 , that is, we show that $\mathcal{A X}$ is a parametrization of $A T(\mathbb{D})$ and $\left(\mathcal{A X}, d_{A S}\right)$ is topologically equivalent to $\left(A T(\mathbb{D}), d_{A T}\right)$, where $d_{A T}$ is the Teichmüller metric on $A T(\mathbb{D})$.

Remark 2. Let $e_{j}^{p}$ be an edge in $\mathcal{F}$ with tip $p$ and let $a_{j}^{p}$ be the endpoint of $e_{j}^{p}$ not equal to $p$. Note first that $\min \left\{g\left(e_{m+k}^{p}\right), g\left(e_{m-k}^{p}\right)\right\} \rightarrow \infty$ is equivalent to say that the minimal scale of the corresponding quadruple $\left\{p, a_{m-k}^{p}, a_{m}^{p}, a_{m+k}^{p}\right\} \rightarrow 0$. From the definitions of $d_{A S}$ and $d_{A C}$, it follows that for any two points $\left[h_{1}\right]$ and $\left[h_{2}\right]$ in $A T(\mathbb{D})$,

$$
d_{A S}\left(\left[s_{1}\right],\left[s_{2}\right]\right) \leq d_{A C}\left(\left[h_{1}\right],\left[h_{2}\right]\right) .
$$

From Theorem 7 , we know that $d_{A T}\left(\left[h_{n}\right],[h]\right) \rightarrow 0$ implies $d_{A S}\left(\left[s_{n}\right],[s]\right) \rightarrow$ 0 as $n \rightarrow \infty$. Our Theorem 2 states that $d_{A T}\left(\left[h_{n}\right],[h]\right) \rightarrow 0$ if and only if $d_{A S}\left(\left[s_{n}\right],[s]\right) \rightarrow 0$ as $n \rightarrow \infty$.

Remark 3. If $h_{2}$ is the identity map, then $s_{2} \equiv 0$ and $s_{2}(p ; m, k) \equiv 1$. Thus

$$
d_{A S}\left(s_{1}, 0\right)=\sup _{p \in \mathcal{P}}\left\{\limsup _{\min \left\{g\left(e_{m+k}^{p}\right), g\left(e_{m-k}^{p}\right)\right\} \rightarrow \infty}\left|\log s_{1}(p ; m, k)\right|\right\} .
$$

It has already been proved in Theorem $A$ of [21] that $h_{1}$ is symmetric if and only if $d_{A S}\left(s_{1}, 0\right)=0$. This means the equivalence class containing the 
Characterization of Asymptotic Teichmüller Space Through Shears 539

identity map is the collection $S\left(\mathbb{S}^{1}\right)$ of all symmetric homeomorphisms in $Q S\left(\mathbb{S}^{1}\right)$. This is a special case of our Theorem 1 .

Proof of Theorem 1. Given two points $h$ and $h^{\prime}$ in $T(\mathbb{D})$, let $s$ and $s^{\prime}$ be the shear maps of $\widetilde{h}=A^{-1} \circ h \circ A$ and $\widetilde{h}^{\prime}=A^{-1} \circ h^{\prime} \circ A$ respectively. If $[h]=$ $\left[h^{\prime}\right]$, then it follows from Remark 2 and Theorem 7 that $d_{A S}\left(s, s^{\prime}\right)=0$.

In the following, we apply some ideas in [21] to show that $d_{A S}\left(s, s^{\prime}\right)=0$ implies $[h]=\left[h^{\prime}\right]$. Suppose on the contrary this is not true. Then there exist two points $h$ and $h^{\prime}$ in $T(\mathbb{D})$ such that $d_{A S}\left(s, s^{\prime}\right)=0$ but $[h] \neq\left[h^{\prime}\right]$. Using the same notation in the previous section, we let $A=-\frac{z-i}{z+i}$ and $\widetilde{h}=A^{-1}$ 。 $h \circ A$ and $\widetilde{h}^{\prime}=A^{-1} \circ \widetilde{h}^{\prime} \circ A$. Denote by $\operatorname{ex}(\widetilde{h})$ and $e x\left(\widetilde{h^{\prime}}\right)$ the Douady-Earle extensions of $\widetilde{h}$ and $\widetilde{h^{\prime}}$ respectively. Since $[h] \neq\left[h^{\prime}\right]$, there exist a constant $c>0$ and a sequence of points $z_{n} \in \mathbb{H}$ leaving every compact subset of $\mathbb{H}$ such that

$$
\left|\mu_{e x(\widetilde{h})}\left(z_{n}\right)-\mu_{e x\left(\widetilde{h^{\prime}}\right)}\left(z_{n}\right)\right| \geq c .
$$

Denote by $\triangle_{n}$ the ideal triangle in $\mathcal{F}$ such that $z_{n} \in \bar{\triangle}_{n}$ and by $A_{n}$ the element in $P S L(2, \mathbb{Z})$ such that $A_{n}\left(\triangle_{n}\right)=\triangle_{0}$. Let $B_{n}$ and $B_{n}^{\prime}$ be the elements in $\operatorname{PSL}(2, \mathbb{R})$ such that $\widetilde{h}_{n}=B_{n} \circ \widetilde{h} \circ\left(A_{n}\right)^{-1}$ and $\widetilde{h}_{n}^{\prime}=B_{n}^{\prime} \circ \widetilde{h}^{\prime} \circ\left(A_{n}\right)^{-1}$ fixing 0,1 and $\infty$.

Let $z_{n}^{\prime}=A_{n}\left(z_{n}\right)$. It suffices to consider the following two cases: one is that $\left\{z_{n}^{\prime}\right\}_{n=1}^{\infty}$ has a subsequence staying in a compact set of $\bar{\triangle}_{0}$ and the other is that $z_{n}$ has a subsequence converging to one of the endpoint of $\triangle_{0}$ as $n \rightarrow \infty$. In the second case, without loss of generality we assume that $z_{n}$ converges to $\infty$ as $n \rightarrow \infty$.

In the first case, for brevity in notation we continue to denote by $\left\{z_{n}^{\prime}\right\}_{n=1}^{\infty}$ the subsequence staying in a compact set of $\bar{\triangle}_{0}$. Given an edge $e \in \mathcal{F}$, we claim that the Farey generation $g\left(A_{n}^{-1}(e)\right)$ goes to $\infty$ as $n \rightarrow \infty$. This is clear if $e$ is an edge $e_{0}$ of $\triangle_{0}$ (since $A_{n}^{-1}\left(e_{0}\right)$ is an edge of $\left.\triangle_{n}\right)$. For any other edge $e \in \mathcal{F}$, we note that for any $n \in \mathbb{N}$,

$$
g\left(A_{n}^{-1}(e)\right)-g\left(A_{n}^{-1}\left(e_{0}\right)\right)=g(e)-g\left(e_{0}\right),
$$

which implies that $g\left(A_{n}^{-1}(e)\right) \rightarrow \infty$ as $n \rightarrow \infty$. Now using the assumption $d_{A S}\left(s, s^{\prime}\right)=0$, we know for each $e \in \mathcal{F}, s \circ A_{n}^{-1}(e)$ and $s_{\widetilde{h}^{\prime}} \circ A_{n}^{-1}(e)$ converge to the same limiting value. Therefore, $\left\{\widetilde{h}_{n}\right\}_{n=1}^{\infty}$ and $\left\{\widetilde{h}_{n}^{\prime}\right\}_{n=1}^{\infty}$ converge to the same limiting homeomorphism pointwisely. Since $\left\{K\left(\operatorname{ex}\left(\widetilde{h}_{n}\right)\right)\right\}_{n=1}^{\infty}$ and $\left\{K\left(\operatorname{ex}\left(\widetilde{h}_{n}^{\prime}\right)\right)\right\}_{n=1}^{\infty}$ are bounded and all $\tilde{h}_{n}$ and $\tilde{h}_{n}^{\prime}$ are normalized to fix three points, passing to subsequences we may assume that $\left\{\widetilde{h}_{n}\right\}_{n=1}^{\infty}$ and $\left\{\widetilde{h}_{n}^{\prime}\right\}_{n=1}^{\infty}$ 
converge uniformly to the same quasisymmetric homeomorphism of $\widehat{\mathbb{R}}$. Thus,

$$
\left|\mu_{e x(\widetilde{h})}\left(z_{n}\right)-\mu_{e x\left(\widetilde{h}^{\prime}\right)}\left(z_{n}\right)\right|=\left|\mu_{e x\left(\widetilde{h}_{n}^{\prime}\right)}\left(z_{n}^{\prime}\right)-\mu_{e x\left(\widetilde{h}_{n}\right)}\left(z_{n}^{\prime}\right)\right| \rightarrow 0
$$

as $n \rightarrow \infty$. This is a contradiction to (5.1).

In the second case, let $\lambda_{n, 1}$ be the greatest integer less than or equal to $\operatorname{Im}\left(z_{n}^{\prime}\right)$. Then $\lambda_{n, 1} \rightarrow \infty$ as $n \rightarrow \infty$. Let $\beta_{n, 1}$ and $\beta_{n, 1}^{\prime}$ be the two real numbers such that $\widehat{h}_{n, 1}(x)=\beta_{n, 1} \widetilde{h}_{n}\left(\lambda_{n, 1} x\right)$ and $\widehat{h}_{n, 1}^{\prime}(x)=\beta_{n, 1}^{\prime} \widetilde{h}_{n}^{\prime}\left(\lambda_{n, 1} x\right)$ fix 0,1 and $\infty$.

Let $e_{k}^{\infty}$ be the edge in $\mathcal{F}$ connecting an integer $k$ to $\infty$. At first we claim that there exists at most one $k_{0} \in \mathbb{Z}$ such that the Farey generation $g\left(A_{n}^{-1}\left(e_{\lambda_{n, 1} k_{0}}^{\infty}\right)\right)$ is bounded as $n \rightarrow \infty$. In the fan $\left\{e_{k}^{\infty}\right\}_{k \in \mathbb{Z}}$, there are $\left|\lambda_{n, 1} k_{0}-\lambda_{n, 1} k\right|$ edges between $e_{\lambda_{n, 1} k_{0}}^{\infty}$ and $e_{\lambda_{n, 1} k}^{\infty}$. Given any two integers $k \neq k_{0}$, since $\left|\lambda_{n, 1} k_{0}-\lambda_{n, 1} k\right| \rightarrow \infty$ as $n \rightarrow \infty$, it follows that $g\left(A_{n}^{-1}\left(e_{\lambda_{n, 1}}^{\infty}\right)\right)$ is unbounded if $g\left(A_{n}^{-1}\left(e_{\lambda_{n, 1} k_{0}}^{\infty}\right)\right)$ is bounded. Thus, the claim follows.

We now show that for all $k \in \mathbb{Z}$,

$$
\lim _{n \rightarrow \infty} \widehat{h}_{n, 1}(k)=\lim _{n \rightarrow \infty} \widehat{h}_{n, 1}^{\prime}(k) .
$$

We divide the proof into three cases according to the value of $k_{0}: k_{0}=0$, $k_{0}<0$ or $k_{0}>0$.

If $k_{0}=0$, then the Farey generations $g\left(A_{n}^{-1}\left(e_{-\lambda_{n, 1}}^{\infty}\right)\right)$ and $g\left(A_{n}^{-1}\left(e_{\lambda_{n, 1}}^{\infty}\right)\right)$ go to $\infty$ as $n \rightarrow \infty$. Since $d_{A S}\left(s, s^{\prime}\right)=0$, it follows from Remarks 1 and 2 that

$$
\begin{aligned}
& \frac{s \circ A_{n}^{-1}\left(\infty, 0, \lambda_{n, 1}\right)}{s^{\prime} \circ A_{n}^{-1}\left(\infty, 0, \lambda_{n, 1}\right)}=\frac{\operatorname{cr}\left(\widetilde{h}_{n}^{\prime}\left(-\lambda_{n, 1}, 0, \lambda_{n, 1}, \infty\right)\right)}{\operatorname{cr}\left(h_{n}\left(-\lambda_{n, 1}, 0, \lambda_{n, 1}, \infty\right)\right)} \\
& =\frac{\operatorname{cr}\left(\widehat{h}_{n, 1}^{\prime}(-1,0,1, \infty)\right)}{\operatorname{cr}\left(\widehat{h}_{n, 1}(-1,0,1, \infty)\right)}=\frac{\widehat{h}_{n, 1}^{\prime}(-1)}{\widehat{h}_{n, 1}(-1)} \rightarrow 1 \text { as } n \rightarrow \infty
\end{aligned}
$$

Thus,

$$
\lim _{n \rightarrow \infty} \widehat{h}_{n, 1}(-1)=\lim _{n \rightarrow \infty} \widehat{h}_{n, 1}^{\prime}(-1) .
$$

Using the symmetric triple $(-1,1,3)$, the assumption $\widehat{h}_{n, 1}(1)=\widehat{h}_{n, 1}^{\prime}(1)$ and the equality of the above two limits, we first obtain

$$
\lim _{n \rightarrow \infty} \widehat{h}_{n, 1}(3)=\lim _{n \rightarrow \infty} \widehat{h}_{n, 1}^{\prime}(3) .
$$

Then we use $(1,2,3)$ and then $(2,3,4)$ and so on to obtain that

$$
\lim _{n \rightarrow \infty} \widehat{h}_{n, 1}(k)=\lim _{n \rightarrow \infty} \widehat{h}_{n, 1}^{\prime}(k)
$$


for each $k \in \mathbb{N}$. Thirdly, for each $k \in \mathbb{N}$, we use the triple $(-k, 0, k)$ to obtain that

$$
\lim _{n \rightarrow \infty} \widehat{h}_{n, 1}(-k)=\lim _{n \rightarrow \infty} \widehat{h}_{n, 1}^{\prime}(-k) .
$$

Using the normalization condition, it is clear that

$$
\lim _{n \rightarrow \infty} \widehat{h}_{n, 1}(0)=\lim _{n \rightarrow \infty} \widehat{h}_{n, 1}^{\prime}(0) .
$$

Therefore, (5.2) holds if $k_{0}=0$.

If $k_{0}<0$, we first use the triple $(0,1,2)$ to obtain

$$
\lim _{n \rightarrow \infty} \widehat{h}_{n, 1}(2)=\lim _{n \rightarrow \infty} \widehat{h}_{n, 1}^{\prime}(2) .
$$

Using the argument to handle the case when $k_{0}=0$, we obtain for each $k \in \mathbb{N}$,

$$
\lim _{n \rightarrow \infty} \widehat{h}_{n, 1}(k)=\lim _{n \rightarrow \infty} \widehat{h}_{n, 1}^{\prime}(k) .
$$

Thirdly, for each $k \in \mathbb{Z} \backslash k_{0}$, we use $(-k, 0, k)$ to obtain that

$$
\lim _{n \rightarrow \infty} \widehat{h}_{n, 1}(k)=\lim _{n \rightarrow \infty} \widehat{h}_{n, 1}^{\prime}(k) .
$$

Finally, we use $\left(k_{0}-1, k_{0}, k_{0}+1\right)$ to obtain that

$$
\lim _{n \rightarrow \infty} \widehat{h}_{n, 1}\left(k_{0}\right)=\lim _{n \rightarrow \infty} \widehat{h}_{n, 1}^{\prime}\left(k_{0}\right) .
$$

Therefore, we show (5.2) if $k_{0}<0$.

The proof of $(5.2)$ for $k_{0}>0$ is very similar to show it for $k_{0}<0$. We skip it.

For each $r \in \mathbb{N}$, let $\lambda_{n, r}$ be the greatest integral multiple of $2^{r-1}$ less than or equal to $\operatorname{Im}\left(z_{n}^{\prime}\right)$. Let $\beta_{n, r}$ and $\beta_{n, r}^{\prime}$ be the real numbers such that $\widehat{h}_{n, r}(x)=$ $\beta_{n, r} \widetilde{h}_{n}\left(\lambda_{n, r} x\right)$ and $\widehat{h}_{n, r}^{\prime}(x)=\beta_{n, r}^{\prime} \widetilde{h}_{n}\left(\lambda_{n, r} x\right)$ fix 0,1 and $\infty$. Using the same argument in the case when $r=1$, it is easy to see that there exists at most one integer $k_{r-1} \in \mathbb{Z}$ such that the Farey generation $g\left(A_{n}^{-1}\left(e_{\lambda_{n, r} k_{r-1}}^{\infty}\right)\right)$ is bounded as $n \rightarrow \infty$. Now we claim that for each $r \in \mathbb{N}$ and each $k \in \mathbb{Z}$,

$$
\lim _{n \rightarrow \infty} \widehat{h}_{n, r}\left(\frac{k}{2^{r-1}}\right)=\lim _{n \rightarrow \infty} \widehat{h}_{n, r}^{\prime}\left(\frac{k}{2^{r-1}}\right) .
$$

This claim can be proved by an induction on $r$. We have just shown the case when $r=1$. Assume that the claim is true for $r=m$, we only need to show the claim is true for $r=m+1$ and $k$ is odd. Given each $k \in \mathbb{Z}$, we can 
choose an odd integer $k^{\prime} \in \mathbb{Z}$ such that both $\frac{k-k^{\prime}}{2^{m-1}}$ and $\frac{k+k^{\prime}}{2^{m-1}}$ are not equal to $k_{r-1}$. Since the limits of $\widehat{h}_{n, r}$ and $\widehat{h}_{n, r}^{\prime}$ are the same at the points $\frac{k-k^{\prime}}{2^{m-1}}$ and $\frac{k-k^{\prime}}{2^{m-1}}$, using the symmetric triple $\left(\frac{k-k^{\prime}}{2^{m-1}}, \frac{k}{2^{m}}, \frac{k+k^{\prime}}{2^{m-1}}\right)$ we obtain the claim 5.3 for $r=m+1$ and an odd integer $k$.

For each $r \in \mathbb{N}$, since $\left\{K\left(\operatorname{ex}\left(\widehat{h}_{n, r}\right)\right)\right\}_{n=1}^{\infty}$ and $\left\{K\left(\operatorname{ex}\left(\widehat{h}_{n, r}^{\prime}\right)\right)\right\}_{n=1}^{\infty}$ are bounded and all the maps in these two sequences are normalized to fix three points, passing to subsequences we may assume that $\left\{\widehat{h}_{n, r}\right\}_{n=1}^{\infty}$ and $\left\{\widehat{h}_{n, r}^{\prime}\right\}_{n=1}^{\infty}$ converge uniformly to two quasisymmetric homeomorphisms of $\widehat{\mathbb{R}}$, which agree on $\left\{\frac{k}{2^{r-1}}: k \in \mathbb{Z}\right\}$ because of the previous claim 5.3 . Then for each $r \in \mathbb{N}$, we choose a sufficiently big $n_{r}$ such that for each $k \in \mathbb{Z}$,

$$
\left|\widehat{h}_{n_{r}, r}\left(\frac{k}{2^{r-1}}\right)-\widehat{h}_{n_{r}, r}^{\prime}\left(\frac{k}{2^{r-1}}\right)\right| \leq 1 / r .
$$

Since $\operatorname{Im}\left(z_{n_{r}}^{\prime}\right) \rightarrow \infty$ as $n \rightarrow \infty$, we can also choose $n_{r}$ large enough such that $1 \leq \operatorname{Im}\left(z_{n_{r}}^{\prime}\right) / \lambda_{n_{r}, r}<2$. Then $\left\{\frac{z_{n_{r}}^{\prime}}{\lambda_{n_{r}, r}}\right\}_{r=1}^{\infty}$ stays in a compact subset of $\mathbb{H}$. Because $\left\{\frac{k}{2^{r}}: k \in \mathbb{Z}, r \in \mathbb{N}\right\}$ is dense in $\widehat{\mathbb{R}}$, the two sequences $\left\{\widehat{h}_{n_{r}, r}\right\}_{r=1}^{\infty}$ and $\left\{\widehat{h}_{n_{r}, r}^{\prime}\right\}_{r=1}^{\infty}$ converge pointwisely, and hence converge uniformly, to the same quasisymmetric homeomorphism of $\widehat{\mathbb{R}}$. Using Proposition 1, we conclude that

$$
\left|\mu_{e x\left(\widehat{h}_{n_{r}, r}\right)}\left(\frac{z_{n_{r}}^{\prime}}{\lambda_{n_{r}, r}}\right)-\mu_{e x\left(\widehat{h}_{n_{r}, r}^{\prime}\right)}\left(\frac{z_{n_{r}}^{\prime}}{\lambda_{n_{r}, r}}\right)\right| \rightarrow 0 \text { as } r \rightarrow \infty .
$$

On the other hand, using (5.1) we obtain for each $r \in \mathbb{N}$,

$$
\begin{aligned}
\left|\mu_{e x\left(\widehat{h}_{n_{r}, r}\right)}\left(\frac{z_{n_{r}}^{\prime}}{\lambda_{n_{r}, r}}\right)-\mu_{\operatorname{ex}\left(\widehat{h}_{n_{r}, r}^{\prime}\right)}\left(\frac{z_{n_{r}}^{\prime}}{\lambda_{n_{r}, r}}\right)\right| & =\left|\mu_{\operatorname{ex}\left(\widetilde{h}_{n_{r}, r}\right)}\left(z_{n_{r}, r}^{\prime}\right)-\mu_{\operatorname{ex}\left(\widetilde{h}_{n_{r}, r}^{\prime}\right)}\left(z_{n_{r}, r}^{\prime}\right)\right| \\
& =\left|\mu_{\operatorname{ex}(\widetilde{h})}\left(z_{n_{r}, r}\right)-\mu_{\operatorname{ex}\left(\widetilde{h^{\prime}}\right)}\left(z_{n_{r}, r}\right)\right| \\
& \geq c>0 .
\end{aligned}
$$

This is a contradiction to (5.4).

Proof of Theorem 2. By Theorem 7 and Remark 2, we see that $d_{A T}\left(\left[h_{n}\right],[h]\right) \rightarrow 0$ implies $d_{A S}\left(\left[s_{n}\right],[s]\right) \rightarrow 0$ as $n \rightarrow \infty$.

It remains to show that $d_{A S}\left(\left[s_{n}\right],[s]\right) \rightarrow 0$ implies $d_{A T}\left(\left[h_{n}\right],[h]\right) \rightarrow 0$ as $n \rightarrow \infty$. Suppose that on the contrary this implication is not true. Then there exist $[h] \in A T(\mathbb{D})$ and a sequence $\left\{\left[h_{n}\right]\right\}_{n=1}^{\infty}$ in $A T(\mathbb{D})$ such that $d_{A S}\left(\left[s_{n}\right],[s]\right)$ goes to 0 but $d_{A T}\left(\left[h_{n}\right],[h]\right)$ dose not go to 0 as $n \rightarrow \infty$. Passing to a subsequence, we may assume that

$$
d_{A T}\left(\left[h_{n}\right],[h]\right) \geq c^{\prime}
$$


Characterization of Asymptotic Teichmüller Space Through Shears 543

for some $c^{\prime}>0$ and all $n$. Then there exist a constant $c>0$ and a sequence of points $z_{n} \in \mathbb{H}$ leaving every compact subset of $\mathbb{H}$ such that

$$
\left|\mu_{e x(\widetilde{h})}\left(z_{n}\right)-\mu_{e x\left(\widetilde{h}_{n}\right)}\left(z_{n}\right)\right| \geq c .
$$

Using a very similar strategy in the proof of the previous theorem, a contradiction can be derived as follows.

Denote by $\triangle_{n}$ the ideal triangle in $\mathcal{F}$ such that $z_{n} \in \bar{\triangle}_{n}$ and by $A_{n}$ the element in $\operatorname{PSL}(2, \mathbb{Z})$ such that $A_{n}\left(\triangle_{n}\right)=\triangle_{0}$. Let $B_{n}$ and $B_{n}^{\prime}$ be the elements in $\operatorname{PSL}(2, \mathbb{R})$ such that $\widehat{h}_{n}=B_{n} \circ \widetilde{h} \circ\left(A_{n}\right)^{-1}$ and $\widehat{h}_{n}^{\prime}=B_{n}^{\prime} \circ \widetilde{h}_{n} \circ$ $\left(A_{n}\right)^{-1}$ fix 0,1 and $\infty$. Let $z_{n}^{\prime}=A_{n}\left(z_{n}\right)$. Consider the following two cases: one is that the sequence $\left\{z_{n}^{\prime}\right\}_{n=1}^{\infty}$ has a subsequence staying in a compact set of $\bar{\triangle}_{0}$ and the other is that without loss of generality, we may assume $z_{n}$ goes to $\infty$ as $n \rightarrow \infty$.

In the first case, we denote the subsequence by $\left\{z_{n}^{\prime}\right\}_{n=1}^{\infty}$ again. For each $e \in \mathcal{F}, g\left(A_{n}^{-1}(e)\right)$ goes to $\infty$ as $n \rightarrow \infty$. Using the assumption that $d_{A S}\left(\left[s_{n}\right],[s]\right)$ approaches 0 as $n \rightarrow \infty$, we see that $s \circ A_{n}^{-1}(e)$ and $s_{n} \circ A_{n}^{-1}(e)$ converge to the same limit for each $e \in \mathcal{F}$. Using the normalization condition, we conclude that $\left\{\widehat{h}_{n}\right\}_{n=1}^{\infty}$ and $\left\{\widehat{h}_{n}^{\prime}\right\}_{n=1}^{\infty}$ converge pointwisely, and hence converge uniformly, to the same quasisymmetric homeomorphism of $\widehat{\mathbb{R}}$. Using Proposition 1, we obtain

$$
\left|\mu_{e x\left(\widehat{h}_{n}^{\prime}\right)}\left(z_{n}^{\prime}\right)-\mu_{e x\left(\widehat{h}_{n}\right)}\left(z_{n}^{\prime}\right)\right| \rightarrow 0 \text { as } n \rightarrow \infty .
$$

On the other hand, (5.5) implies

$$
\left|\mu_{e x\left(\widehat{h}_{n}^{\prime}\right)}\left(z_{n}^{\prime}\right)-\mu_{e x\left(\widehat{h}_{n}\right)}\left(z_{n}^{\prime}\right)\right|=\left|\mu_{e x(\widetilde{h})}\left(z_{n}\right)-\mu_{e x\left(\widetilde{h}_{n}\right)}\left(z_{n}\right)\right| \geq c>0 .
$$

This is a contradiction to (5.6).

In the second case, for each $r \in \mathbb{N}$, let $\lambda_{n, r}$ be the greatest integral multiple of $2^{r-1}$ less than or equal to $\operatorname{Im}\left(z_{n}^{\prime}\right)$. Let $\beta_{n, r}$ and $\beta_{n, r}^{\prime}$ be the real numbers such that $\widehat{h}_{n, r}(x)=\beta_{n, r} \widetilde{h}_{n}\left(\lambda_{n, r} x\right)$ and $\widehat{h}_{n, r}^{\prime}(x)=\beta_{n, r}^{\prime} \widetilde{h}_{n}\left(\lambda_{n, r} x\right)$ fix 0,1 and $\infty$. Using the assumption that $d_{A S}\left(\left[s_{n}\right],[s]\right) \rightarrow 0$ as $n \rightarrow \infty$ and following the exact same details used to derive a contradiction for the second case in the proof of Theorem 11, we can conclude that for each $r \in \mathbb{N}$, $\left\{\widehat{h}_{n, r}\right\}_{n=1}^{\infty}$ and $\left\{\widehat{h}_{n, r}^{\prime}\right\}_{n=1}^{\infty}$ converge pointwisely to two quasisymmetric homeomorphisms of $\widehat{\mathbb{R}}$ that agree on the set $\left\{\frac{k}{2^{r-1}}, k \in \mathbb{Z}\right\}$. Then the part of the last paragraph in the proof of Theorem 1 after the first sentence also forms the rest of the proof for Theorem 2 . 


\section{References}

1. W. Abikoff, Conformal barycenters and the Douady-Earle extension - A discrete dynamical approach, Jour. d'Analyse Math. 86 (2002) 221-234.

2. A. Douady and C. Earle, Conformally natural extension of homeomorphisms of the circle, Acta Math. 157 (1986) 23-48.

3. C. J. Earle, F. P. Gardiner, N. Lakic, Asymptotic Teichmüller space. I. The complex structure, In the tradition of Ahlfors and Bers, 17-38, Contemp. Math. 256, Amer. Math. Soc., Providence, RI, 2000.

4. C. J. Earle, F. P. Gardiner, N. Lakic, Asymptotic Teichmüller space. II. The metric structure, In the tradition of Ahlfors and Bers, III, 187-219, Contemp. Math. 355, Amer. Math. Soc., Providence, RI, 2004.

5. C. J. Earle, V. Markovic, D. Šarić, Barycentric extension and the Bers embedding for asymptotic Teichmüller space, In Complex manifolds and hyperbolic geometry (ed. C. J. Earle, W. J. Harvey and S. RecillasPishmish), 87-105, Contemp. Math. 311, Amer. Math. Soc., Providence, RI, 2002.

6. J. Fan and J. Hu, Topological characterization of an asymptotic Teichmüller space through measured geodesic laminations, Preprint, Graduate Center of CUNY, 2013.

7. F. P. Gardiner, J. Hu and N. Lakic, Earthquake curves, In Complex Manifolds and Hyperbolic Geometry (ed. C. J. Earle, W. J. Harvey and S. Recillas-Pishmish), 141-195, Contemp. Math. 311, Amer. Math. Soc., Providence, RI, 2002.

8. F. P. Gardiner, N. Lakic, Quasiconformal Teichmüller spaces, Math Surveys and Monogr. 76, Amer. Math. Soc., (2000).

9. F. P. Gardiner, D. Sullivan, Symmetric structures on a closed curve, Amer. J. Math. 114 (1992) 683-736.

10. J. Hu, Earthquake measure and cross-ratio distortion, In the tradition of Ahlfors and Bers, III, 285-308, Contemp. Math., 355, Amer. Math. Soc., Providence, RI, 2004.

11. J. Hu and O. Muzician, Cross-ratio distortion and Douady-Earle extension: I. A new upper bound on quasiconformality, Jour. of London Math. Soc. 86(2) (2012) 387-406. 
Characterization of Asymptotic Teichmüller Space Through Shears 545

12. J. Hu and O. Muzician, Cross-ratio distortion and Douady-Earle extension: II. Quasiconformality and asymptotic conformality are local, Jour. d'Analyse Math. 117(1) (2012) 249-271.

13. J. Hu and O. Muzician, Cross-ratio distortion and Douady-Earle extension: III. How the maximal dilatation of the extension near the origin is controlled by the distortion of the boundary map on finitely many points, Preprint, 2014.

14. J. Hu and O. Muzician, Conformally natural extensions of continuous circle maps: I. The case when the pushforward measure has no atom, Contemp. Math. 575 (2012) 171-198.

15. J. Hu and O. Muzician, Conformally natural extensions of continuous circle maps: II. The general case, to appear in Jour. d'Analyse Math..

16. J. Hu and S. Pal, Boundary differentiability of Doudy-Earle extensions of diffeomorphisms of $\mathbb{S}^{n}$, to appear in Pure and Applied Mathematics Quarterly.

17. V. Markovic, Quasisymmetric groups, J. Amer. Math. Soc. 19 (2006) 673-715.

18. H. Miyachi and D. Šarić, Uniform Weak* Topology and earthquake in the hyperbolic plane, Proc. London Math. Soc. 105 (2012) 1123-1148.

19. R. C. Penner, Universal constructions in Teichmüller theory, Adv. in Math. 98 (1993) 143-215.

20. D. Šarić, Real and complex earthquakes, Trans. Amer. Math. Soc. 358 (2006) 233-249.

21. D. Šarić, Circle homeomorphisms and shears, Geom. Topol. 14 (2010) 2405-2430.

22. D. Šarić, Zygmund vector fields, Hilbert transformation and Fourier coefficients in shear coordinates, Amer. J. Math. 135(6) (2013) 1559-1600.

23. W. Thurston, Earthquakes in two-dimensional hyperbolic geometry, In Low-dimensional topology and Kleinian groups, LMS. Lecture Note Ser. 112, Cambridge Univ. Press, Cambridge (1986) 91-112.

24. C. Wu, The cross-ratio distortion of integrably asymptotic affine homeomorphism of unit circle, Sci. China Math. 55 (2012) 625-632.

25. S. Wu, Moduli of quadrilaterals and extremal quasiconformal extensions of quasisymmetric functions, Comment. Math. Helv. 72 (1997) 593-604. 
Jinhua Fan

Department of Applied Mathematics

Nanjing University of Science and Technology

Nanjing 210094, PRC

E-mail: jinhuafan@hotmail.com

Jun $\mathrm{Hu}$

Department of Mathematics

Brooklyn College of CUNY

Brooklyn, NY 11210

and

Ph.D. Program in Mathematics

Graduate Center of CUNY

365 Fifth Avenue, New York, NY 10016

E-mail: junhu@brooklyn.cuny.edu or JHu1@gc.cuny.edu 